\title{
miR-17-5p promotes the invasion and migration of colorectal cancer by regulating HSPB2
}

\section{Weifang Yu}

The First Hospital of Hebei Medical University

Jia Wang

The First Hospital of Hebei Medical University

Chao Li

The First Hospital of Hebei Medical University

Mingda Xuan

Hebei Medical University

Shuangshuang Han

The First Hospital of Hebei Medical University

\section{Yingfu Zhang}

Hebei Medical University

\section{Pengfei Liu}

Hebei Medical University

Zengren Zhao ( $\sim$ jiangxia0925@hebmu.edu.cn )

The First Hospital of Hebei Medical University

\section{Research Article}

Keywords: MicroRNA (miRNA), colorectal cancer, tumor progression

Posted Date: May 12th, 2021

DOI: https://doi.org/10.21203/rs.3.rs-63587/v3

License: (c) (1) This work is licensed under a Creative Commons Attribution 4.0 International License.

Read Full License 


\section{Abstract}

MicroRNA (miRNA) can affect tumor progression by regulating cell proliferation, apoptosis and metastasis. A significant upregulation of miR-17-5p expression was found in colorectal cancer (CRC) tissues by miRNA microarray chip analysis. However, the underlying mechanism of miR-17-5p in CRC is still unclear. The mRNA expression of miR-17-5p was significantly higher in CRC tissues than in adjacent normal tissues. In CRC group, the expression of miR-17-5p in cancer tissues with lymph node metastasis was higher compared with those without lymph node metastasis. The biological function of miR-17-5p was demonstrated through CCK-8, colony formation, flow cytometry and transwell assays.

Overexpression of miR-17-5p inhibited CRC cell apoptosis, as well as promoting proliferation, migration and invasion. Transcriptome sequencing and miRNA target prediction software suggested that HSPB2 might be a target gene of miR-17-5p and luciferase reporter detection validated for the first time that miR17-5p binds directly to the 3'-UTR of HSPB2. In the rescue experiment, the tumor suppressive effect of HSPB2 was detected and miR-17-5p could promote cell proliferation, migration and invasion by targeting HSPB2. Taken together, miR-17-5p promotes invasion and migration by inhibiting HSPB2 in CRC, thereby implicating its potential as a novel diagnostic biomarker and therapeutic target for CRC.

\section{Introduction}

Colorectal cancer (CRC) is the third common malignancy wordwide associated with increased morbidity and mortality ${ }^{1}$. Five-year survival rate for patients with early CRC is $90 \%$, as compared with $12 \%$ for advanced $\mathrm{CRC}^{2}$. Although several oncogenes and tumor suppressor genes have been reported to be related to $\mathrm{CRC}$, the molecular mechanism of $\mathrm{CRC}$ development remains poorly understood.

MicroRNAs (miRNA) are non-coding RNA species, generally 19-22 nucleotides in length, and can induce translation inhibition or cause mRNA degradation by specifically binding to the target gene ${ }^{3-6}$. It has been reported that miRNA regulates multiple vital cellular processes, including cell growth, differentiation, cycle progression, and apoptosis, and can be an essential biomarker for diagnosis and treatment of tumors ${ }^{7-10}$. miR-17-5p, a member of the miR-17-92 cluster, is located in the third intron on chromosome 13 , which is an amplified genomic site in many cancers ${ }^{11,12}$. Previously, miR-17-5p was found to be abnormally expressed in multiple cancers and is involved in the tumorigenesis and progression by inhibiting apoptosis and promoting proliferation and metastasis ${ }^{13-20}$. The previous analysis of miRNA microarray chips by our team has revealed a significant upregulation of miR-17-5p expression in CRC tissues than adjacent normal tissues ${ }^{21}$. However, the precise molecular mechanism through which miR17-5p influences CRC progression remains largely unclear.

In this study, the expression level of miR-17-5p in CRC and adjacent normal tissues were analyzed, and its biological effects on CRC cells were investigated. To our knowledge, this is the first report to confirm that HSPB2, as a downstream target gene of miR-17-5p, plays an oncogenic miRNA role in the pathogenesis of $\mathrm{CRC}$ by regulating HSPB2. These findings suggest its potential as a novel diagnostic biomarker and target for future $\mathrm{CRC}$ treatments. 


\section{Results}

miR-17-5p was upregulated in most of the CRC tissues and cells. We first examined the expression of miR-17-5p in 47 paired samples of CRC and the adjacent normal tissues. We found that miR-17-5p levels was significantly upregulated in CRC tissues compared to adjacent normal tissues (Fig. 1A), which was consistent with our previous results by microarray chips in 4 pairs of CRC tissues. In the CRC group, the expression of miR-17-5p in cancer tissues with lymph node metastasis was higher compared to those without lymph node metastasis (Fig. 1B), but it was not associated with clinicopathological features such as degree of differentiation, patient age, sex, tumor location and invasion (Table 1). In addition, high expression of miR-17-5p was found in most CRC cell lines (Fig. 1C).

\section{Overexpression of miR-17-5p inhibited CRC cell apoptosis, and promoted proliferation, invasion and} migration. To determine whether miR-17-5p overexpression modulated CRC tumorigenesis, HCT116 cells with a certain miR-17-5p expression were transfected with miR-17-5p mimic, and the overexpression effect was confirmed by qRT-PCR (Fig. 2A). A flow cytometry assay showed that HCT116 cells overexpressing miR-17-5p had a significant reduction of apoptotic rate compared to control group (Fig. 2B), as evident from the CCK8 assay that miR-17-5p overexpression induced the viability of CRC cells (Fig. 2C). And colony formation assay revealed that the number of colonies was increased in cells overexpressing miR-17-5p (Fig. 2D). Moreover, Transwell assay showed that over-expression of miR-17-5p resulted in a significant increase in the number of migrating and invading cells compared with control cells (Fig. 2E).

Silencing of miR-17-5p inhibited CRC cell proliferation, invasion and migration. HCT116 cells were subsequently transfected with inhibitor that could endogenously suppress the expression of miR-17-5p, and a decrease expression of miR-17-5p was confirmed via qRT-PCR (Fig. 3A). Apoptotic rate was not significantly decreased after the expression of miR-17-5p was suppressed, as compared with the control cells (Fig. 3B). The CCK8 assay indicated that silencing endogenous miR-17-5p reduced the viability of CRC cells (Fig. 3C). And colony formation assay showed that the number of colonies in miR-17-5p silenced cells was significantly reduced (Fig. 3D). In addition, the silencing of endogenous miR-17-5p reduced cell migration and invasion compared to the control cells in the Transwell assay (Fig. 3E).

HSPB2 was a direct target of miR-17-5p. In order to gain insight into the mechanism by which miR-17-5p acts as an oncogenic miRNA in CRC cells, we performed a transcriptome sequencing analysis to profile differentially expressed genes in HCT116 infected with miR-17-5p mimic and inhibitor. Significant difference revealed by RNA-seq analysis indicated global alterations in gene expression (Fig. 4A). The mRNA expression of 647 and 419 genes was altered by overexpression or knockdown of miR-17-5p, respectively, of which 27 genes were regulated by both (Fig. 4B). Many upregulated or downregulated genes might also be indirect targets, as they were affected as part of the regulatory network. Then we used three bioinformatics algorithms (TargetScan, microRNA.org, and mirtarbase) to screen whether there were potential miR-17-5p binding sites in these 27 genes. We found HSPB2 and miR-17-5p had opposite expression pattern changes according to the transcriptome sequencing analysis and qRT-PCR (Fig. 4C-D). 
In addition, there are potential binding sites for miR-17-5p in the 3'-UTR region of HSPB2 to regulate translation or expression (Fig. 5A). Therefore, these findings suggested that HSPB2 was a strong candidate for a direct and functional target gene of miR-17-5p in CRC. Then a luciferase reporter gene assay was performed using the firefly reporter plasmids containing the binding site for miR-17-5p. Compared to control group, the overexpression of miR-17-5p resulted in a significant reduction in luciferase reporter activity at three sites (Fig. 5B), whereas miR-17-5p inhibitor led to a significant increase in reporter activity (Fig. 5C).

The HSPB2 mRNA was downregulated in CRC. We analyzed the expression levels of HSPB2 mRNA in 51 paired CRC and adjacent normal tissues. The results showed that the expression of HSPB2 in CRC tissues was significantly reduced compared to normal adjacent tissues (Fig. 6A). Moreover, compared with normal tissues, the expression of HSPB2 mRNA in cancer tissues was significantly reduced according to the CRC dataset of TCGA, the COAD and READ datasets of GEPIA (Fig. 6B-D).

Interaction mechanism of miR-17-5p and HSPB2 in CRC. Next, we performed a rescue experiment in HCT116 and LOVO cells. When miR-17-5p expression was exogenously increased, we verified that overexpressed miR-17-5p could enhance cell viability, colony formation (Fig. 7C-D), migration and invasion (Fig. 8) as compared with the control group. When miR-17-5p was endogenously silenced, it was reconfirmed that compared to the control group, knockdown of miR-17-5p could reduce cell viability, colony formation (Fig. 9C-D), migration, and invasion (Fig. 10). We overexpressed HSPB2 in HCT116 and LOVO (Fig. 7A-B). CCK8 and colony formation assay revealed that HSPB2 overexpression inhibited CRC cell viability and colony formation compared to the control group (Fig. 7C-D). The Transwell assay showed that overexpression of HSPB2 significantly reduced the number of migrating and invading cells compared to the control group (Fig. 8). Then HSPB2 expression was knocked down (Fig. 9A-B). Compared with the control cells, reduced HSPB2 promoted cell viability and colony formation (Fig. 9C-D), and increased the number of migrating and invading cells (Fig. 10). In addition, we found that overexpression of miR-17-5p suppressed HSPB2 at both the mRNA and protein levels (Fig. 7A-B) and silencing of endogenous miR-17-5p increased HSPB2 at both the mRNA and protein levels (Fig. 9A-B). When miR-17-5p and HSPB2 expression were increased simultaneously, the reintroducing HSPB2 caused by co-overexpression attenuated the tumor-promoting effect of overexpressed miR-17-5p, as shown by reduced proliferation (Fig. 7C-D), migration and invasion (Fig. 8). Simultaneous knockdown of miR-17-5p and HSPB2 revealed that reduced HSPB2 could partially reverse the suppression of proliferation (Fig. 9CD), migration and invasion (Fig. 10) caused by miR-17-5p inhibitor.

\section{Discussion}

In 2018, the International Agency for Research on Cancer (IARC) estimated that there would be more than 521,000 new cases and more than 248,000 new deaths of CRC in China, making it the major public health issue $^{22}$. The clinical process of CRC is caused by multi-level interactions ${ }^{23}$, among which the genetic changes of oncogenes or tumor suppressor genes are the most important drivers of cancer occurrence and development. MiRNAs are short endogenous non-coding RNA that can regulate the expression of 
related genes at the post-transcriptional level ${ }^{24}$. And it has been reported that some miRNAs could exert a vital influence on pathogenesis of $\mathrm{CRC}^{25-31}$. We previously used microarray chip analysis to screen differentially expressed miRNAs in 4 pairs of CRC tissues. It was found that the expression of miR-17-5p was increased in CRC compared to normal tissues ${ }^{21}$, suggesting that miR-17-5p had the potential to promote CRC tumorigenesis. The effects that miR-17-5p could exert on tumorigenesis and CRC development, as well as the downstream target genes and interaction mechanisms of miR-17-5p are our prime concerns.

miR-17-5p was reported to be a critical oncogenic miRNA, and the increased expression of miR-17-5p was found to be involved in the development of multiple tumors, including hepatocellular carcinoma ${ }^{15}$, gastric cancer $^{32}$, prostate cancer ${ }^{33}$, ovarian cancer ${ }^{34}$, and breast cancer ${ }^{35}$. However, miR-17-5p overexpression was also found to inhibit cell proliferation, invasion, and tumor metastasis in certain cancer cell lines ${ }^{36}$. In this study, we detected the expression of miR-17-5p in 47 pairs of CRC tissue and adjacent normal tissue samples, and the results showed that miR-17-5p was significantly upregulated in CRC tissues and was associated with lymph node metastasis. Moreover, we found that overexpression of miR-17-5p promoted the proliferation, anti-apoptosis, migration and invasion of CRC cells, whereas knockdown of miR-17-5p inhibited cell proliferation, migration and invasion but had no significant effect on apoptosis. Taken together, these results suggest that miR-17-5p may contribute to the development of CRC by promoting certain malignant biological characteristics such as growth and metastasis.

In order to investigate the target genes of miR-17-5p in CRC, we first performed transcriptome sequencing to obtain differentially expressed genes affected by expression of miR-17-5p. It was noteworthy that 647 differentially expressed genes were affected by miR-17-5p overexpression, 419 differentially expressed genes regulated by miR-17-5p knockdown, and 27 differentially expressed genes regulated by both. To further focus on potential target genes, we used three bioinformatics algorithms (TargetScan, microRNA.org, and mirtarbase) to predict the potential binding site for miR-17-5p in these 27 genes. We identified HSPB2 as a strong candidate gene based on the above data. Subsequently, the luciferase reporter assay was used to validate that miR-17-5p directly binds to the 3'-UTR region of HSPB2 in two CRC cell lines. To our knowledge, HSPB2 was proposed for the first time as a direct target gene of miR-17$5 p$.

HSPB2, a novel and unique member of the Heat shock proteins (HSPs) family, is located on the 11q22q23 chromosome ${ }^{37,38}$. HSPs can interact with its substrate to keep the denatured protein in a folded state and transform it into a functional conformation as a molecular chaperone $\mathrm{e}^{39,40}$. Furthermore, HSPs have been reported to be involved in cancer cell proliferation, survival, and apoptosis ${ }^{41-43}$. Studies have shown that expression of HSPB2 is reduced in esophageal squamous cell carcinoma ${ }^{44}$ and pancreatic cancer, which can inhibit the progression of pancreatic cancer ${ }^{45}$. HSPB2 also inhibits the apoptotic pathway by inhibiting the activation of caspases-8 in breast cancer ${ }^{46}$. Moreover, studies have revealed that HSPB2 is a novel target of $p 53^{47}$, which can restore the transcriptional activity of mutant p53 and inhibit tumor progression to a certain extent ${ }^{45}$. However, there have been no reports to date concerning the effect of 
HSPB2 on CRC as a downstream of miR-17-5p. We analyzed the expression of HSPB2 mRNA in 51 paired samples of CRC and adjacent normal tissues, and the results showed that the expression of HSPB2 in $\mathrm{CRC}$ was significantly reduced. Because no studies have reported the expression of HSPB2 in CRC, we checked the expression of HSPB2 in the network data set. Compared to normal tissues, the expression of HSPB2 in cancer tissues was significantly reduced according to the CRC dataset of TCGA, the COAD and READ datasets of GEPIA. This suggests the potential role of HSPB2 as a CRC suppressor gene and makes our study clinically relevant. In subsequent rescue experiments, the biological function of HSPB2 was tested in two CRC cell lines (HCT116 and LOVO). The results showed that overexpressed HSPB2 inhibited cell proliferation, migration and invasion in CRC. To our knowledge, we showed for the first time that HSPB2 inhibits the progression of CRC.

To further verify the direct relationship between miR-17-5p and HSPB2 and the underlying mechanism, a rigorous rescue experiment was performed. When miR-17-5p was overexpressed or silenced, HSPB2 expression was decreased or increased at the mRNA and protein levels. This suggests that miR-17-5p inhibits expression of HSPB2 in CRC cells and a strong miRNA-mRNA interaction. Next, we cooverexpressed miR-17-5p and HSPB2, and subsequent biological functional tests revealed that reintroducing HSPB2 could partially reverse the miR-17-5p-mediated promotion of cell proliferation, migration, and invasion. After simultaneous knockdown of miR-17-5p and HSPB2, as expected, reduced HSPB2 was rescued by miR-17-5p inhibitors from suppressing tumors such as proliferation, migration, and invasion. Taken together, our findings uncover a novel mechanism for the first time through which miR-17-5p inhibits its expression through the miR-17-5p-HSPB2 axis, thereby promoting CRC tumorigenesis by inhibiting HSPB2, a downstream target of miR-17-5p.

In summary, this study unravels a novel mechanism for tumor progression through which miR-17-5p promotes CRC cell proliferation, migration and invasion by targeting HSPB2. This suggests that miR-17$5 p$ can be used as a diagnostic and prognostic biomarker for CRC patients. Future studies on miR-17-5p and HSPB2 are warranted to develop novel molecular therapeutic avenues.

\section{Materials And Methods}

Patients and specimens. Ninety-eight paired specimens of CRC tissues and adjacent normal tissues were provided by Clinical Biobank in the First Hospital of Hebei Medical University. Written informed consent was obtained from all patients. CRC and normal tissues were collected from CRC resection specimens, and none of patients had received radiotherapy or chemotherapy prior to surgery. Study protocols were approved by the Ethics Committee of the First Hospital of Hebei Medical University and adhered to the principles of the Declaration of Helsinki.

Cell lines culture. Human CRC cell lines HCT116, SW480, LOVO, SW1116, Caco2, and SW1463 cell lines were obtained from Prof. Jun Yu (The Chinese University of Hong Kong, Hong Kong, China). HCT116 were cultured in McCoy's 5A medium (Gibco, Gaithersburg, MD, USA) and the other cells lines were cultured in DMEM medium (Gibco, Gaithersburg, MD, USA, supplemented with $10 \%$ fetal bovine serum 
(FBS; FBS; Gibco, Gaithersburg, MD, USA) and 1\% penicillin-streptomycin (Invitrogen, Carlsbad, CA, USA) in a $37^{\circ} \mathrm{C}$ humidified incubator with $95 \%$ air and $5 \% \mathrm{CO}_{2}$.

Transfection and plasmid construction. The hsa-miR-17-5p mimic, inhibitor, HSPB2 overexpression plasmid or shRNA-HSPB2 and a respective negative control (GeneCopoeia, MD, USA) were transfected into cells in 6-well plates using Lipofectamine 2000 (Invitrogen, Carlsbad, USA) when the cells were approximately $60-80 \%$ confluent. After the cells were cultured in medium without FBS for $6 \mathrm{~h}$, the medium was replaced by that supplemented with $10 \%$ FBS. Cells were harvested $48 \mathrm{~h}$ after transfection and the effect of transfection was assessed by real-time quantitative polymerase chain reaction (qRT$\mathrm{PCR})$.

RNA extraction and qRT-PCR. Total RNA was extracted from the transfected cells and tissues using Trizol Reagent (Invitrogen, Carlsbad, USA). To quantify miR-17-5p, All-in-One miRNA qRT-PCR kits (GeneCopoeia, MD, USA) were used with miR-17-5p-specific primers (HmiRQP0230, GeneCopoeia, MD, USA) according to the manufacturer's instructions. To quantify HSPB2, CDNA was reverse transcribed using PrimeScript RT reagent kit (Takara, Beijing, China). Then, a qRT-PCR was performed by using SYBR Green Master Mix (Vazyme, NJ, USA). U6 was used as internal control of miRNA, and GAPDH was used as internal control of mRNA. The primer sequences were as follows: HSPB2(forward): 5'ATGTCGGGCCGCTCAGTGCC-3'; HSPB2(reverse): 5'-GTCACCTCGTCTGGGGTAAA-3' (22); GAPDH (forward):5'-GAGTCAACGGATTTGGTCGT-3'; GAPDH (reverse) 5'-CATGGGTGGAATCATATTGGA-3'. All the experiments were performed in triplicate on an ABI7500 Sequence Detection System, and the mean cycle threshold (CT) data were obtained. The relative amount normalized to the internal control was calculated with the Eq. $2^{-\triangle \Delta C T}$.

Cell apoptosis assay. The transfected cells were stained using the Annexin V-FITC/ PI Apoptosis Detection Kit (Beyotime, Jiangsu, China) in accordance with the manufacturer's instructions. $1 \times 10^{5}$ cells were analyzed for apoptosis using a flow cytometry (BD, MA, USA), and the percentage of the apoptotic cells was quantified using Cell Quest software.

Cell proliferation assay. The transfected cells were plated at $2 \times 10^{3}$ cells per well in 96 -well plates and incubated overnight in medium supplemented with $10 \%$ FBS. Cell proliferation was measured using a Cell Counting Kit-8 (Dojindo, Tokyo, Japan) at 24,48 , and $72 \mathrm{~h}$ post-transfection following the manufacturer's instruction. The absorbance at $450 \mathrm{~nm}$ was measured using a Promega GloMax Luminescence detector (Promega, MD, USA).

Colony formation assay. $1 \times 10^{3}$ transfected cells were plated in cultured dishes and grown for 2 weeks. Subsequently, the cells were washed twice with phosphate buffer saline (PBS), fixed with $4 \%$ paraformaldehyde and stained with $0.5 \%$ crystal violet for $1 \mathrm{~h}$. The dishes were scanned and the average number of colonies was achieved. 
Transwell invasion and migration assay. Invasion and migration assay were performed using Transwell chambers with 8- $\mu \mathrm{m}$ pores (Corning, MA, USA). For invasion assay, $2 \times 10^{5}$ transfected cells suspended in $500 \mu \mathrm{l}$ serum-free medium were placed in the upper chambers coated with Matrigel, and medium containing $10 \%$ FBS was added to the lower chamber as a chemoattractant. For migration assay, cells in $100 \mu \mathrm{l}$ serum-free medium were added to the upper chambers, and medium containing $10 \%$ FBS was placed in the lower chamber as a chemoattractant. Following incubation at $37^{\circ} \mathrm{C}$ for $24 \mathrm{~h}$, cells on the upper surface of the chamber were gently wiped off with cotton swabs. The cells were stained using a Diff-Quick stain kit according to the manufacturer's protocol and counted blindly (five random fields per chamber).

RNA sequencing and data analysis. Total RNA was extracted from cells transfected with hsa-miR-17-5p mimic or inhibitor and the negative control cells, and then subjected to commercial RNA-sequencing (RNA-seq) analysis (Novogene). Preparation of library and sequencing of transcriptome were performed using NEB Next Ultra RNA Library Prep Kit for Illumina (Novogene, Beijing, China) following the manufacturer's protocols, and the attribute sequence of each sample was added with an index code. The clustering of the index-coded samples was achieved on a cBot Cluster Generation System. After cluster generation, 125-bp paired-end reads to genes were mapped using HTSeq v0.6.0 software. Afterwards, fragments per kilobase of transcript per million fragments mapped (FPKM) were also calculated. Analysis of differential expression was performed using the DEGSeq R package through the one scaling normalized factor. Corrected $\mathrm{P}$ values of 0.005 and log2 (fold changes) of 1 were set as the threshold for significantly differential expression after adjusted using the Benjamini-Hochberg method. Moreover, the hierarchical clustering and heatmaps were generated to show the normalized expression among the samples.

Luciferase reporter assay. Four sets of luciferase reporter plasmids were constructed, and each set of plasmids contained a 60-80 bp fragment of HSPB2 3'-untranslational region (3'-UTR) with a conserved miR-17-5p binding site (Sangon Biotech, Shanghai, China). For the luciferase reporter assays, HCT116 and LOVO cells were cultured in 96-well plates, and each well was co-transfected with firefly luciferase reporter plasmids, miR-17-5p mimic, inhibitor or the negative control using Lipofectamine 2000 (Invitrogen, Carlsbad, USA). The firefly luciferase and renilla luciferase activities were evaluated using the Dual Luciferase Reporter Assay Kit (Promega, MD, USA) following the manufacturer's instructions after transfection for $48 \mathrm{~h}$.

Western blot analysis. Total protein was isolated from the cultured cells using RIPA lysis buffer (Beyotime, Shanghai, China). Proteins were separated by 12\% SDS-PAGE (Bio-Rad) and transferred onto polyvinylidene difluoride membranes (Millipore, MA, USA). The immunoreactive protein bands were detected by the Odyssey Scanning System (Li-Cor, Lincoln, USA) after using antibodies against HSPB2 (Proteintech, Wuhan, China) and $\beta$-actin (Solarbio, Beijing, China).

Databases analysis. We used the online databases TargetScan ${ }^{48}$, microRNA.org ${ }^{49}$ and mirtarbase ${ }^{50}$ to predict target genes of miR-17-5p, and confirmed the specific binding sites for miR-17-5p on the 3'-UTR 
region of the target gene. In addition, the online database The Cancer Genome Atlas (TCGA) and Gene Expression Profiling Ineractive Analysis (GEPIA) were used to further ascertain the mRNA expression of the target gene.

Statistical analysis. The results of normal distribution data are expressed as mean \pm SD and the results of non-normal distribution data are expressed as median and quartile spacing. Student' s t-test, one-way ANOVA and two-way ANOVA were used in this study. All statistical analyses were performed using GraphPad Prism 7 (GraphPad Software, CA, USA) and SPSS 21 (IBM, NY, USA). P $<0.05$ was considered as statistically significant.

\section{Declarations}

\section{Acknowledgement}

We thank Dr. Xia Jiang from General Surgery Laboratory of The First Hospital of Hebei Medical University to provide experiments guidance. This study was supported by grants from The National Natural Science Foundation of China (No. 81572758), Hebei Province Key Research and Development Project (No. 18277741D) and other Hebei Province Projects (No. ZH2018002, LS201701, LS201905, 1387, SGH201501, A201802017, LNB201809, G2019035, LNB201909).

\section{Authors contributions}

Conception and design of study: Weifang Yu and Jia Wang. Performed the experiments: Weifang Yu, Jia Wang, Chao Li, Mingda Xuan, Shuangshuang Han. Data analysis and interpretation: Yingfu Zhang, Pengfei Liu. Manuscript writing: Weifang Yu and Jia Wang. Project supervision: Zengren Zhao. Final approval of manuscript: All authors.

\section{Competing Interests}

The authors declare that they have no conflict of interest.

\section{Availability of data and materials}

The data used and/or analyzed during the present study are available from the corresponding author on reasonable request.

\section{Ethical approval}

The study protocol was approved by the Ethics Committee of the First Hospital of Hebei Medical University and adhered to the principles of the Declaration of Helsinki.

\section{References}


1. Siegel, R. L., Miller, K. D., Jemal, A. \& Cancer Statistics 2017. CA: a cancer journal for clinicians 67, 730, doi:10.3322/caac.21387 (2017).

2. Miller, K. D. et al. Cancer treatment and survivorship statistics, 2016. CA: a cancer journal for clinicians. 66, 271-289 https://doi.org/10.3322/caac.21349 (2016).

3. Bartel, D. P. MicroRNAs: genomics, biogenesis, mechanism, and function. Cell. 116, 281-297 https://doi.org/10.1016/s0092-8674(04)00045-5 (2004).

4. Bartel, D. P. MicroRNAs: target recognition and regulatory functions. Cell. 136, 215-233 https://doi.org/10.1016/j.cell.2009.01.002 (2009).

5. Ahmed, F. E. Role of miRNA in carcinogenesis and biomarker selection: a methodological view. Expert review of molecular diagnostics. 7, 569-603 https://doi.org/10.1586/14737159.7.5.569 (2007).

6. Rupaimoole, R. \& Slack, F. J. MicroRNA therapeutics: towards a new era for the management of cancer and other diseases. Nature reviews. Drug discovery. 16, 203-222 https://doi.org/10.1038/nrd.2016.246 (2017).

7. Bracken, C. P., Scott, H. S. \& Goodall, G. J. A network-biology perspective of microRNA function and dysfunction in cancer. Nature reviews. Genetics. 17, 719-732 https://doi.org/10.1038/nrg.2016.134 (2016).

8. He, L. \& Hannon, G. J. MicroRNAs: small RNAs with a big role in gene regulation. Nature reviews. Genetics. 5, 522-531 https://doi.org/10.1038/nrg1379 (2004).

9. Leung, A. K. \& Sharp, P. A. Function and localization of microRNAs in mammalian cells. Cold Spring Harbor symposia on quantitative biology. 71, 29-38 https://doi.org/10.1101/sqb.2006.71.049 (2006).

10. Bandres, E. et al. Epigenetic regulation of microRNA expression in colorectal cancer. International journal of cancer. 125, 2737-2743 https://doi.org/10.1002/ijc.24638 (2009).

11. Fontana, L. et al. MicroRNAs 17-5p-20a-106a control monocytopoiesis through AML1 targeting and M-CSF receptor upregulation. Nature cell biology. 9, 775-787 https://doi.org/10.1038/ncb1613 (2007).

12. Ota, A. et al. Identification and characterization of a novel gene, C13orf25, as a target for 13q31-q32 amplification in malignant lymphoma. Cancer research. 64, 3087-3095 https://doi.org/10.1158/0008-5472.can-03-3773 (2004).

13. Kanaan, Z. et al. A plasma microRNA panel for detection of colorectal adenomas: a step toward more precise screening for colorectal cancer. Annals of surgery. 258, 400-408 https://doi.org/10.1097/SLA.0b013e3182a15bcc (2013).

14. Yang, B. et al. Identification of microRNAs associated with lymphangiogenesis in human gastric cancer. Clinical \& translational oncology: official publication of the Federation of Spanish Oncology Societies and of the National Cancer Institute of Mexico. 16, 374-379 https://doi.org/10.1007/s12094-013-1081-6 (2014).

15. Shan, S. W. et al. Mature miR-17-5p and passenger miR-17-3p induce hepatocellular carcinoma by targeting PTEN, GalNT7 and vimentin in different signal pathways. Journal of cell science. 126, 
1517-1530 https://doi.org/10.1242/jcs.122895 (2013).

16. Lu, S. et al. Increased expression of microRNA-17 predicts poor prognosis in human glioma. Journal of biomedicine \& biotechnology 2012, 970761, doi:10.1155/2012/970761 (2012).

17. Sand, M. et al. Expression of microRNAs in basal cell carcinoma. The British journal of dermatology. 167, 847-855 https://doi.org/10.1111/j.1365-2133.2012.11022.x (2012).

18. Ohuchida, K. et al. MicroRNA-10a is overexpressed in human pancreatic cancer and involved in its invasiveness partially via suppression of the HOXA1 gene. Annals of surgical oncology. 19, 23942402 https://doi.org/10.1245/s10434-012-2252-3 (2012).

19. Yu, J. et al. MicroRNA miR-17-5p is overexpressed in pancreatic cancer, associated with a poor prognosis, and involved in cancer cell proliferation and invasion. Cancer biology \& therapy. 10, 748757 https://doi.org/10.4161/cbt.10.8.13083 (2010).

20. Gu, J. et al. GFRa2 prompts cell growth and chemoresistance through down-regulating tumor suppressor gene PTEN via Mir-17-5p in pancreatic cancer. Cancer letters. 380, 434-441 https://doi.org/10.1016/j.canlet.2016.06.016 (2016).

21. Zhang, L. J. et al. Differential expressions of microRNAs in rectal adenocarcinoma tissues. Cancer Res Prev Treat. 06, 602-605 (2014).

22. Bray, F. et al. Global cancer statistics 2018: GLOBOCAN estimates of incidence and mortality worldwide for 36 cancers in 185 countries. CA: a cancer journal for clinicians. 68, 394-424 https://doi.org/10.3322/caac.21492 (2018).

23. Markowitz, S. D. \& Bertagnolli, M. M. Molecular origins of cancer: Molecular basis of colorectal cancer. The New England journal of medicine. 361, 2449-2460 https://doi.org/10.1056/NEJMra0804588 (2009).

24. Berindan-Neagoe, I., Pdel, M., Pasculli, C., Calin, G. A. \& B. \& MicroRNAome genome: a treasure for cancer diagnosis and therapy. CA: a cancer journal for clinicians. 64, 311-336 https://doi.org/10.3322/caac.21244 (2014).

25. Cho, W. C. MicroRNAs: potential biomarkers for cancer diagnosis, prognosis and targets for therapy. The international journal of biochemistry \& cell biology. 42, 1273-1281 https://doi.org/10.1016/j.biocel.2009.12.014 (2010).

26. Aqeilan, R. I., Calin, G. A. \& Croce, C. M. miR-15a and miR-16-1 in cancer: discovery, function and future perspectives. Cell death and differentiation. 17, 215-220 https://doi.org/10.1038/cdd.2009.69 (2010).

27. Lin, P. L. et al. MicroRNA-21 promotes tumour malignancy via increased nuclear translocation of beta-catenin and predicts poor outcome in APC-mutated but not in APC-wild-type colorectal cancer. Carcinogenesis. 35, 2175-2182 https://doi.org/10.1093/carcin/bgu110 (2014).

28. Nugent, M., Miller, N. \& Kerin, M. J. Circulating miR-34a levels are reduced in colorectal cancer. Journal of surgical oncology. 106, 947-952 https://doi.org/10.1002/jso.23174 (2012).

29. Schetter, A. J., Okayama, H. \& Harris, C. C. The role of microRNAs in colorectal cancer. Cancer journal (Sudbury, Mass.). 18, 244-252 https://doi.org/10.1097/PPO.0b013e318258b78f (2012). 
30. Valeri, N. et al. MicroRNA-135b promotes cancer progression by acting as a downstream effector of oncogenic pathways in colon cancer. Cancer cell. 25, 469-483

https://doi.org/10.1016/j.ccr.2014.03.006 (2014).

31. Strillacci, A. et al. Loss of miR-101 expression promotes Wnt/beta-catenin signalling pathway activation and malignancy in colon cancer cells. The Journal of pathology. 229, 379-389 https://doi.org/10.1002/path.4097 (2013).

32. Wu, Q. et al. miR-17-5p promotes proliferation by targeting SOCS6 in gastric cancer cells. FEBS letters. 588, 2055-2062 https://doi.org/10.1016/j.febslet.2014.04.036 (2014).

33. Yang, X. et al. Both mature miR-17-5p and passenger strand miR-17-3p target TIMP3 and induce prostate tumor growth and invasion. Nucleic acids research. 41, 9688-9704 https://doi.org/10.1093/nar/gkt680 (2013).

34. Li, L. et al. MiR-17-5p up-regulates YES1 to modulate the cell cycle progression and apoptosis in ovarian cancer cell lines. Journal of cellular biochemistry. 116, 1050-1059 https://doi.org/10.1002/jcb.25060 (2015).

35. Yang, F. et al. miR-17 as a diagnostic biomarker regulates cell proliferation in breast cancer. OncoTargets and therapy. 10, 543-550 https://doi.org/10.2147/ott.s127723 (2017).

36. Hossain, A., Kuo, M. T. \& Saunders, G. F. Mir-17-5p regulates breast cancer cell proliferation by inhibiting translation of AIB1 mRNA. Molecular and cellular biology. 26, 8191-8201 https://doi.org/10.1128/mcb.00242-06 (2006).

37. Prabhu, S., Raman, B., Ramakrishna, T. \& Rao Ch, M. HspB2/myotonic dystrophy protein kinase binding protein (MKBP) as a novel molecular chaperone: structural and functional aspects. PloS one. 7, e29810 https://doi.org/10.1371/journal.pone.0029810 (2012).

38. Shama, K. M. et al. Transient up-regulation of myotonic dystrophy protein kinase-binding protein, MKBP, and HSP27 in the neonatal myocardium. Cell structure and function. 24, 1-4 https://doi.org/10.1247/csf.24.1 (1999).

39. Jacob, P., Hirt, H. \& Bendahmane, A. The heat-shock protein/chaperone network and multiple stress resistance. Plant biotechnology journal. 15, 405-414 https://doi.org/10.1111/pbi.12659 (2017).

40. McArdle, A. \& Jackson, M. J. The role of attenuated redox and heat shock protein responses in the age-related decline in skeletal muscle mass and function. Essays in biochemistry. 61, 339-348 https://doi.org/10.1042/ebc20160088 (2017).

41. Calderwood, S. K. Heat shock proteins and cancer: intracellular chaperones or extracellular signalling ligands? Philosophical transactions of the Royal Society of London. Series B, Biological sciences. 373, https://doi.org/10.1098/rstb.2016.0524 (2018).

42. Shevtsov, M. \& Multhoff, G. Heat Shock Protein-Peptide and HSP-Based Immunotherapies for the Treatment of Cancer. Frontiers in immunology. 7, 171 https://doi.org/10.3389/fimmu.2016.00171 (2016).

43. Wang, C. et al. Heat shock proteins in hepatocellular carcinoma: Molecular mechanism and therapeutic potential. International journal of cancer. 138, 1824-1834 
https://doi.org/10.1002/ijc.29723 (2016).

44. Chang, X., Yamashita, K., Sidransky, D. \& Kim, M. S. Promoter methylation of heat shock protein B2 in human esophageal squamous cell carcinoma. International journal of oncology. 38, 1129-1135 https://doi.org/10.3892/ijo.2011.918 (2011).

45. Yu, Z. et al. Molecular chaperone HspB2 inhibited pancreatic cancer cell proliferation via activating p53 downstream gene RPRM, BAI1, and TSAP6. Journal of cellular biochemistry. 121, 2318-2329 https://doi.org/10.1002/jcb.29455 (2020).

46. Oshita, S. E., Chen, F., Kwan, T., Yehiely, F. \& Cryns, V. L. The small heat shock protein HspB2 is a novel anti-apoptotic protein that inhibits apical caspase activation in the extrinsic apoptotic pathway. Breast cancer research and treatment. 124, 307-315 https://doi.org/10.1007/s10549-010-0735-0 (2010).

47. Liu, S. et al. As a novel p53 direct target, bidirectional gene HspB2/alphaB-crystallin regulates the ROS level and Warburg effect. Biochimica et biophysica acta. 1839, 592-603 https://doi.org/10.1016/j.bbagrm.2014.05.017 (2014).

48. Agarwal, V., Bell, G. W., Nam, J. W. \& Bartel, D. P. Predicting effective microRNA target sites in mammalian mRNAs. eLife. 4, https://doi.org/10.7554/eLife.05005 (2015).

49. Betel, D., Wilson, M., Gabow, A., Marks, D. S. \& Sander, C. The microRNA.org resource: targets and expression. Nucleic acids research. 36, D149-153 https://doi.org/10.1093/nar/gkm995 (2008).

50. Hsu, S. D. et al. miRTarBase: a database curates experimentally validated microRNA-target interactions. Nucleic acids research. 39, D163-169 https://doi.org/10.1093/nar/gkq1107 (2011).

\section{Tables}

Table I. The relationship between the relative expression level of miR-17-5p and clinicopathological variables in 47 patients with CRC. 
Clinicopathological data

Gender

Male

Female

Age】years $\square$

$\geq 68$

$\square 68$

Position

Colon

Rectum

Differentiatad degree

Poorly differentiated

Moderately and Highly differentiated 39

$89.1324(8.1624,10.2390)$

$86 \quad 0.230$

$6.1988(3.1362,10.4831)$
$31 \quad 6.7371(3.1734,10.0152) \quad 233 \quad 0.558$

$167.0274(3.9014,14.7741)$
$237.0274(3.2852,9.7473)$

$22 \quad 5.3080(2.8689,7.7239) \quad 327.50 .218$

$258.8520(3.9014,14.2116)$

Invasive status

Penetrating serosa

Non-penetrating serosa

Lymph node metastasis

Absence

Presence

$29 \quad 7.1514(3.5859,9.7126) \quad 213.50 .929$

$186.1988(3.0022,12.6758)$

$295.5983(2.9601,8.8104) \quad 128.50 .018$ *

$18 \quad 8.9922(5.7549,14.5365)$

\section{Figures}


$\mathbf{a}$

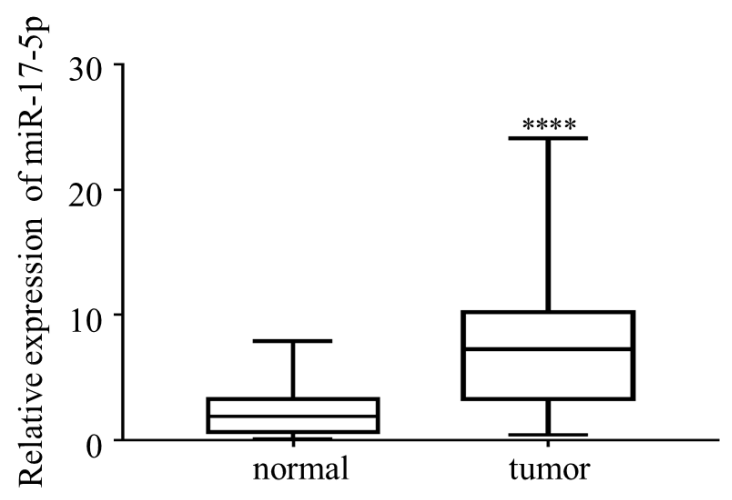

C

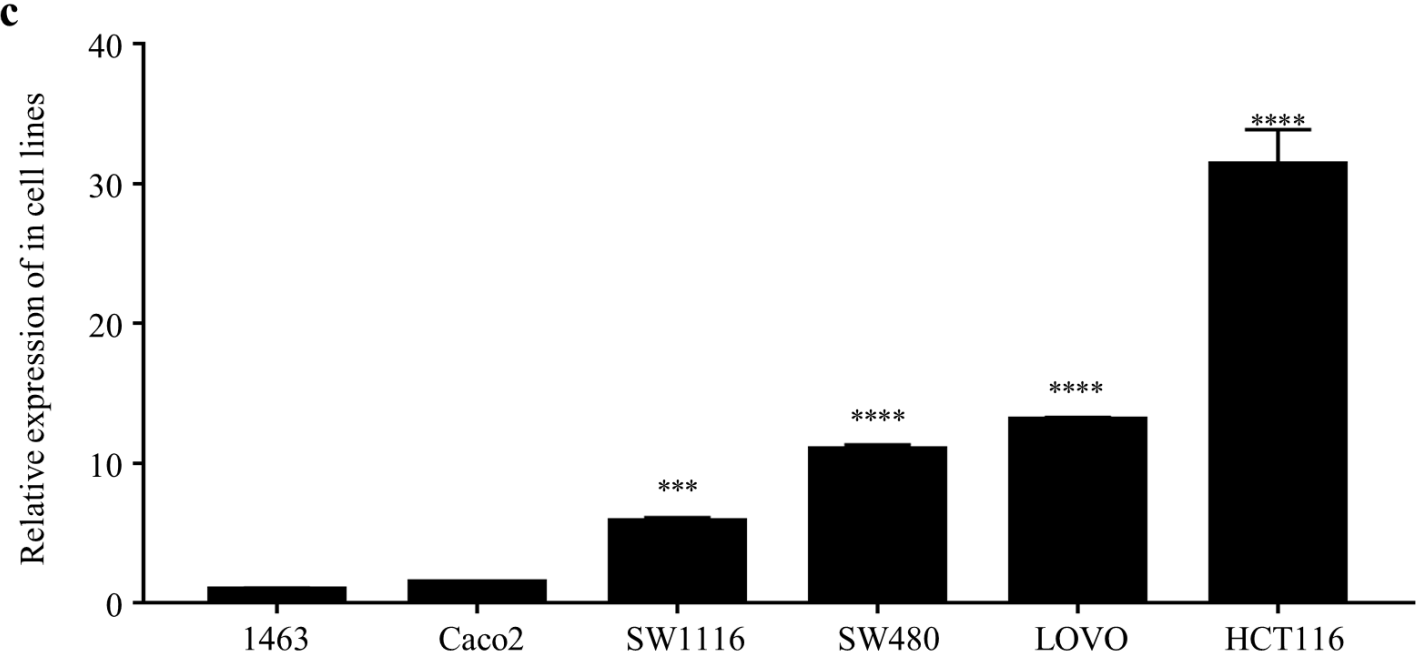

b

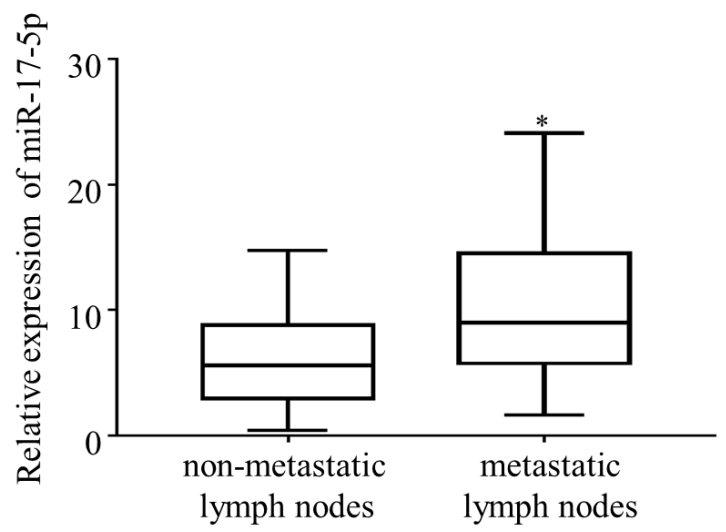

\section{Figure 1}

The expression of miR-17-5p is elevated in CRC patient samples and cell lines. (A) The expression level of miR-17-5p in 47 paired of CRC and adjacent normal tissues by qRT-PCR. (B) qRT-PCR analysis of miR-17$5 p$ expression in CRC tissues with $(n=18)$ and without lymph node metastasis $(n=29)$. (C) Expression levels of miR-17-5p in 6 CRC cell lines. ${ }^{*} P<0.05$; $* \star * ~ P<0.001 ; * \star \star * ~ P<0.0001$. 


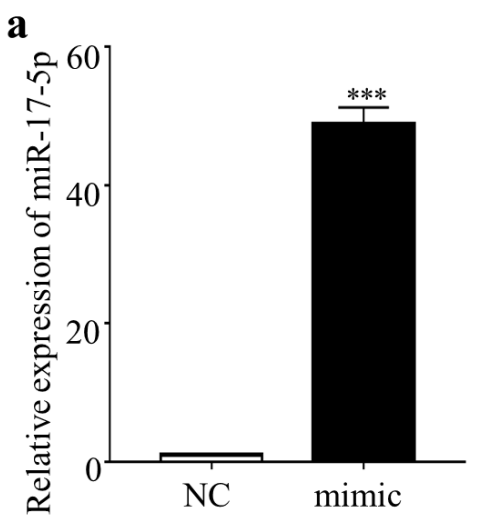

c

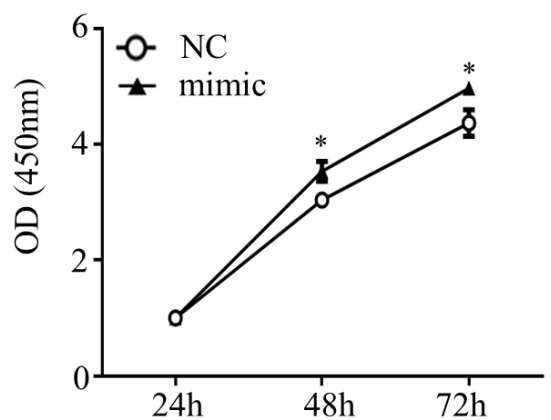

e
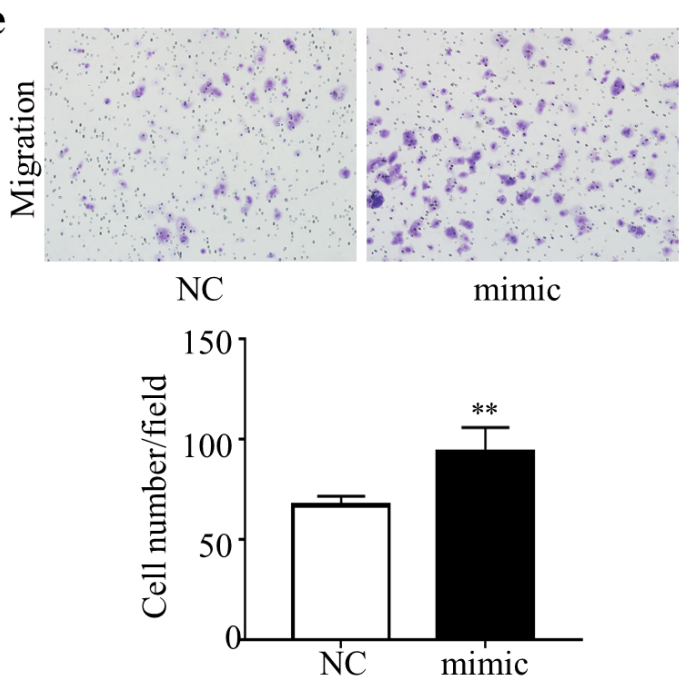
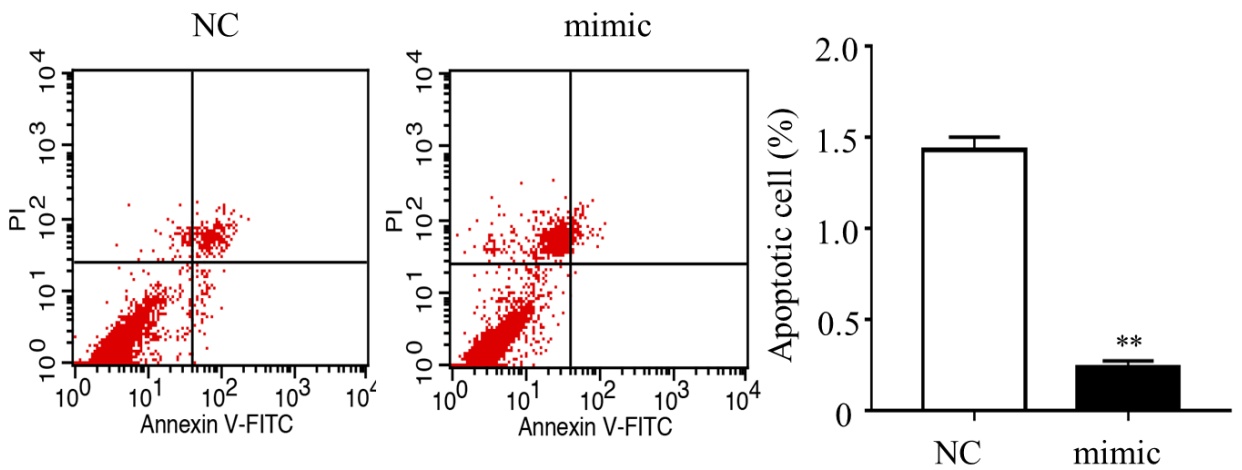

d

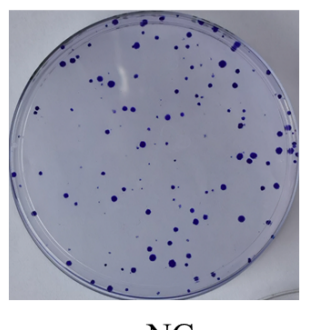

$\mathrm{NC}$

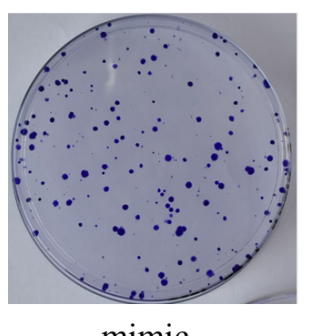

mimic

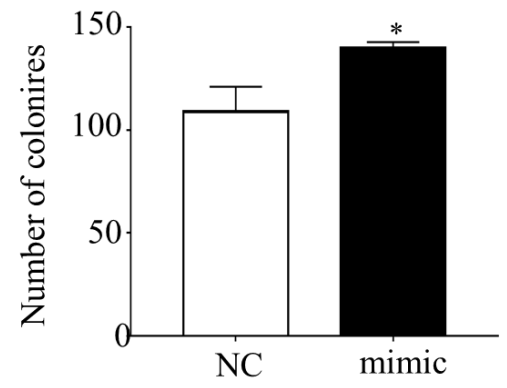

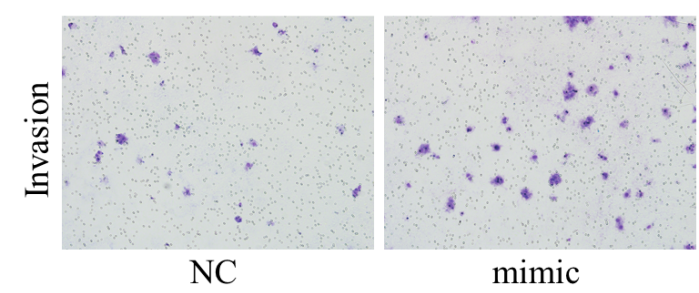

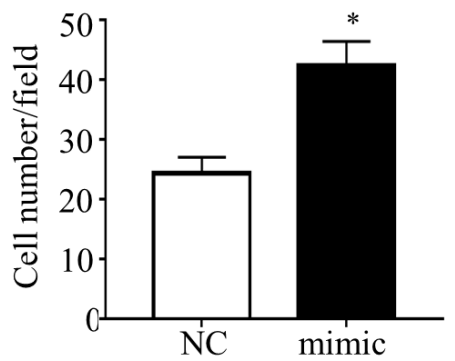

\section{Figure 2}

Overexpressed miR-17-5p inhibited CRC cell apoptosis, promoted the proliferation, invasion and migration. (A) The overexpression miR-17-5p was detected in HCT116 cells by qRT-PCR. (B) Flow cytometry was used to evaluate the cell apoptosis after increasing miR-17-5p expression. (C, D) CCK8 assay and colony formation assay were used to determine the effect of miR-17-5p overexpression on cell proliferation. $(E)$ Transwell assay was performed to assess the migration and invasion potency after the expression of miR-17-5p was up-regulated. ${ }^{*} \mathrm{P}<0.05$; $* \star \mathrm{P}<0.01$; $* \star * \mathrm{P}<0.001$. 


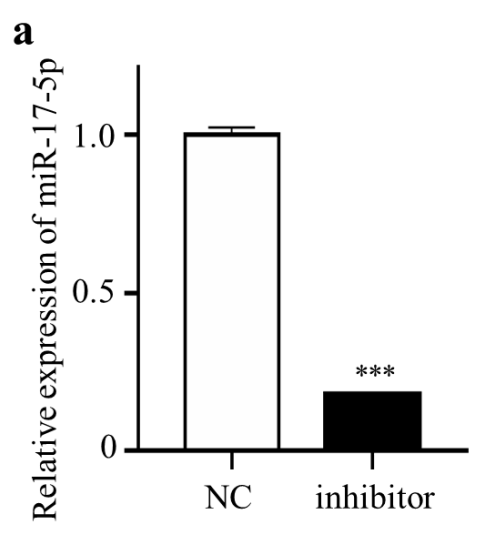

c

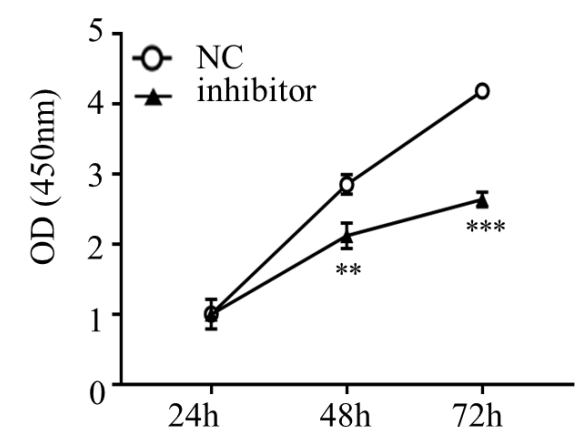

e
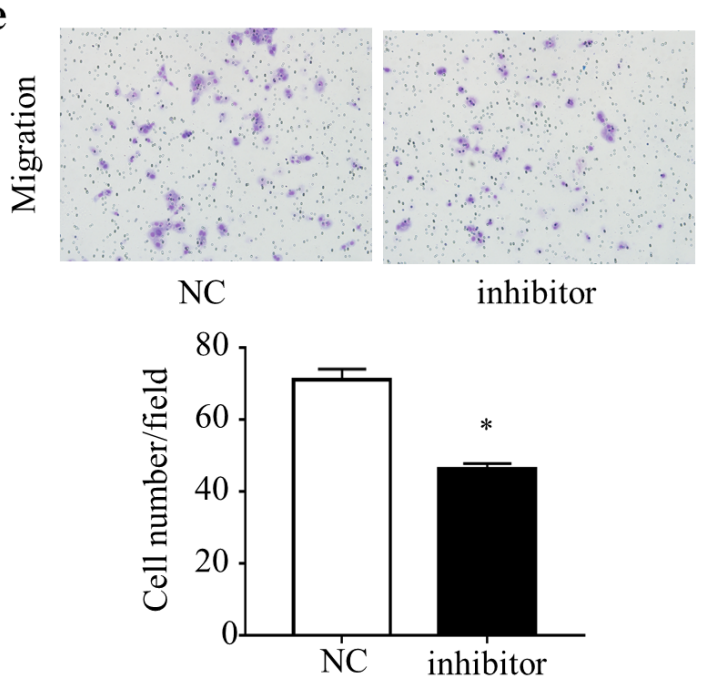

b

d
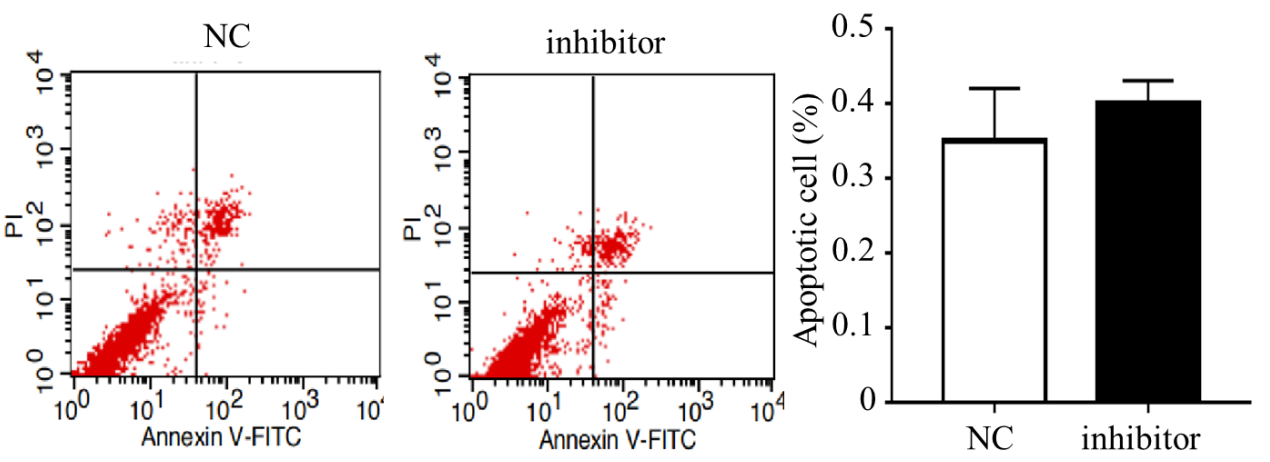

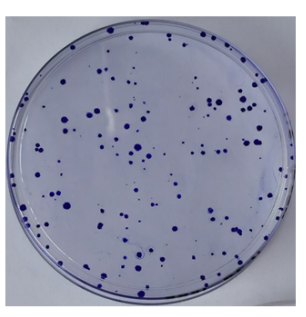

$\mathrm{NC}$

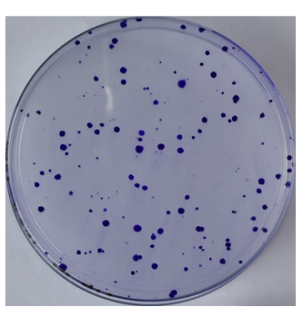

inhibitor
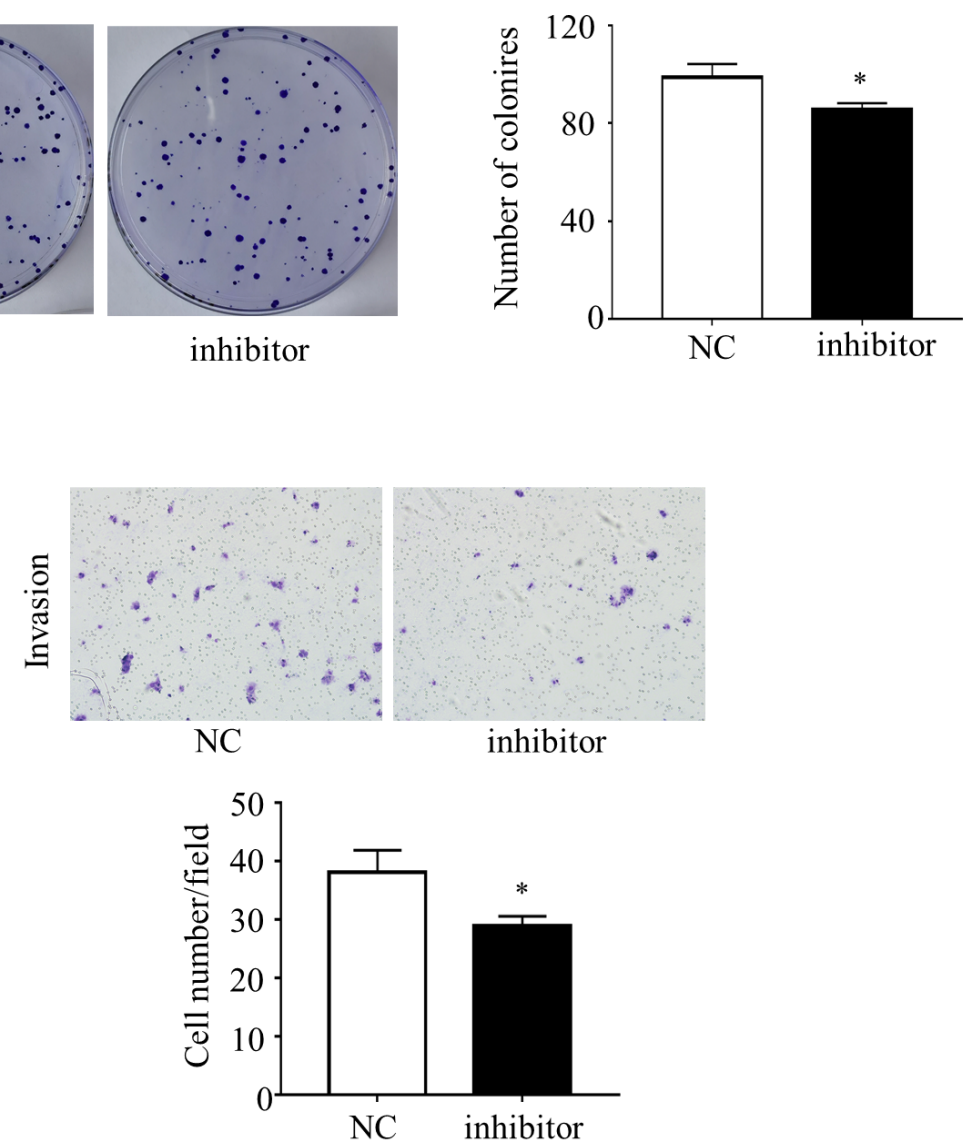

\section{Figure 3}

Overexpressed miR-17-5p inhibited CRC cell apoptosis, promoted the proliferation, invasion and migration. (A) The overexpression miR-17-5p was detected in HCT116 cells by qRT-PCR. (B) Flow cytometry was used to evaluate the cell apoptosis after increasing miR-17-5p expression. (C, D) CCK8 assay and colony formation assay were used to determine the effect of miR-17-5p overexpression on cell proliferation. (E) Transwell assay was performed to assess the migration and invasion potency after the expression of miR-17-5p was up-regulated. ${ }^{*} \mathrm{P}<0.05$; $* \star \mathrm{P}<0.01$; $* \star \star \mathrm{P}<0.001$. 
a

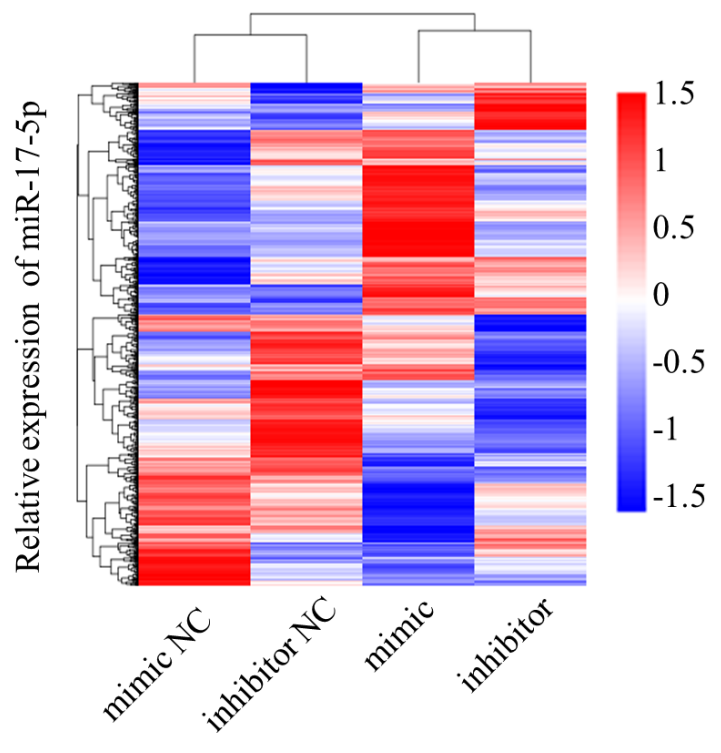

c

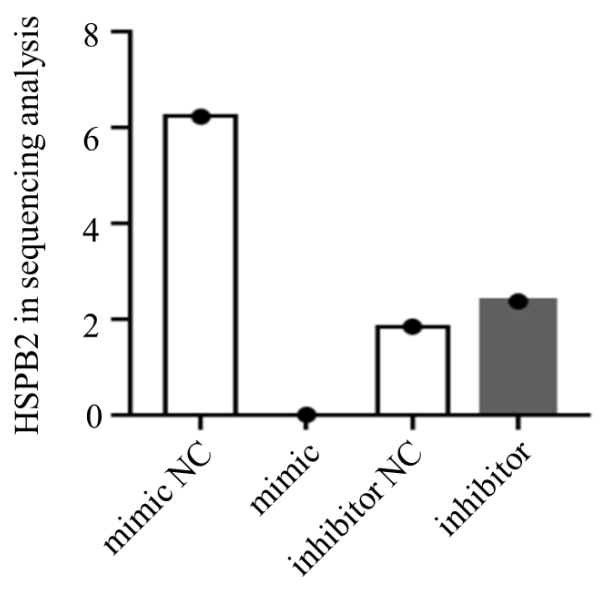

b

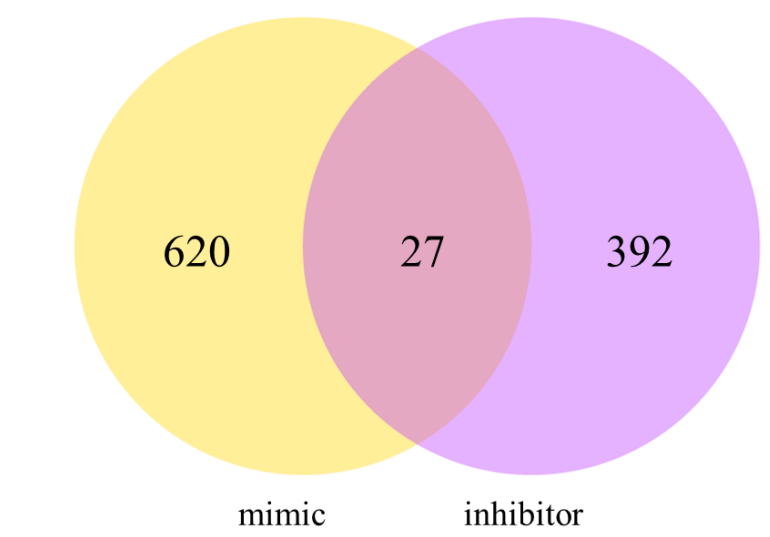

d

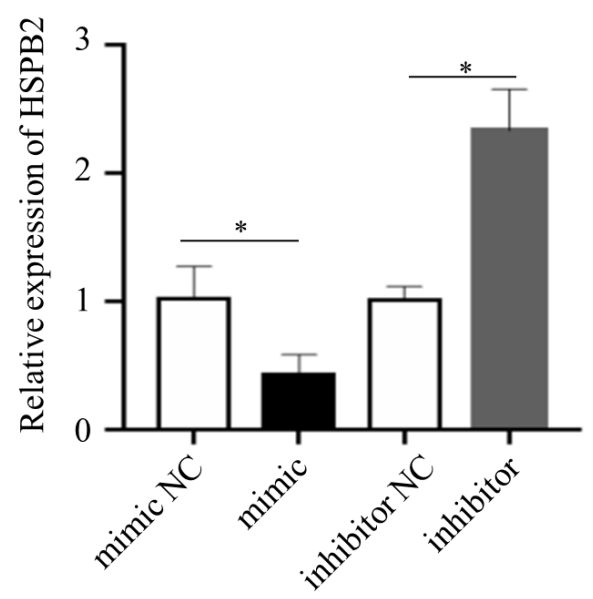

\section{Figure 4}

HSPB2 is predicted to be the target of miR-17-5p. (A) Hierarchical clustering heat map of RNA sequencing show all differential genes in up and down regulated miR-17-5p cells. (B) Venn diagram depicting the overlap differential genes in affected by miR-17-5p overexpression and knockdown. (C) Analysis data of HSPB2 expression in sequencing samples. (D) The relative expression of HSPB2 mRNA in sequencing samples by qRT-PCR. * $\mathrm{P}<0.05$ 
site 1

miR-17-5p 3' GAUGGACGUGACAỤUC-GUGAAAC 5' $:$ I I I I I I: I I I I I I I I HSPB2 5' AUUUCUGGA-UGUGAGCCACUUUA $3^{\prime}$

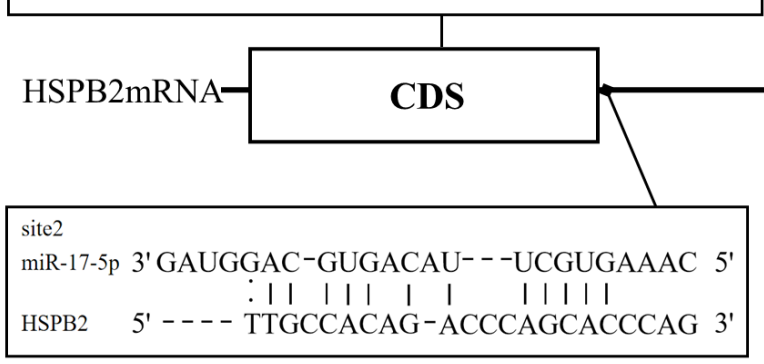

b

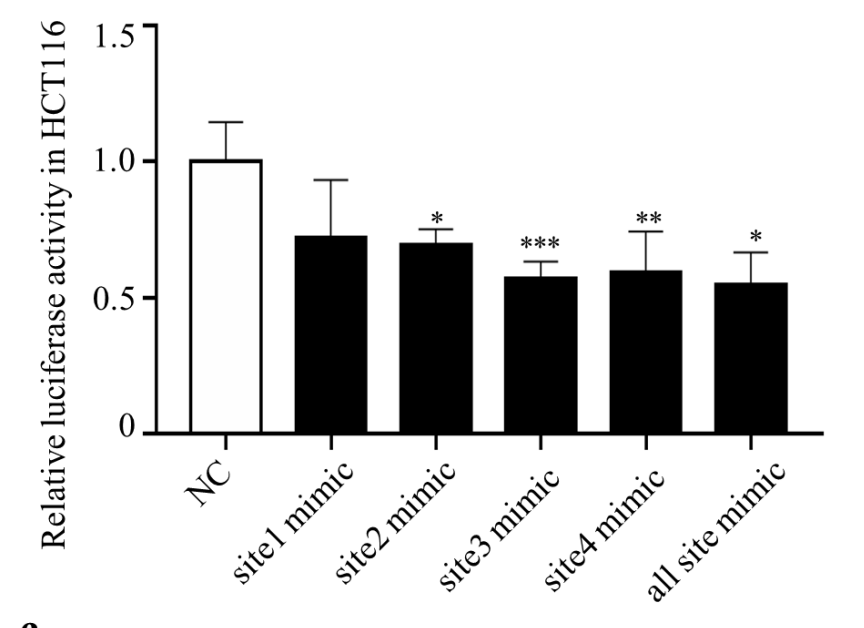

C

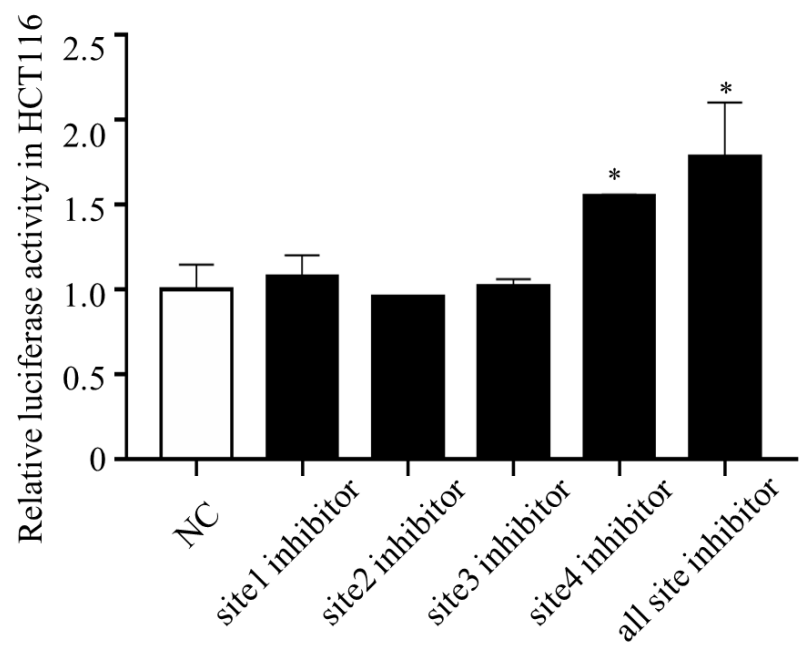

\section{b}

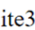

miR-17-5p 3' GAUGGACGUGA- - - - - -CAU - -UCGU- -GAAAC 5' I I I I | |

| I I I | | I | Hspb2 5' TGCCCACCACTCCAGAGGTAGCAGCATCCTTGG 3'

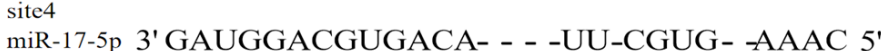

I I I 1: I I I I I:1: I I I

HSPB2 5' AAAGGTGCATGGTCCACAATGTATGGTTTG 3'
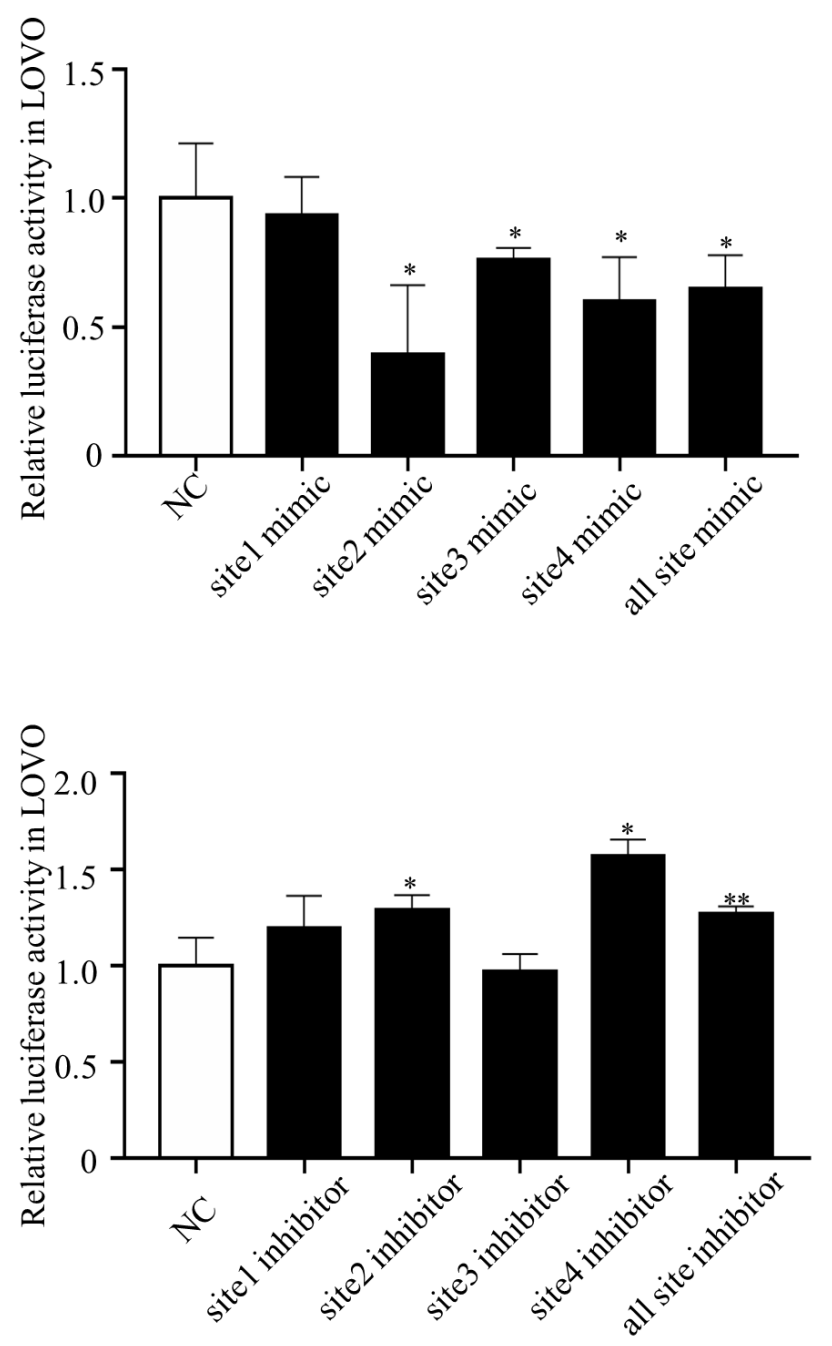

Figure 5

HSPB2 is identified as a direct target of miR-17-5p. (A) Predicted binding sites of miR-17-5p at the 3'-UTR of HSPB2 mRNA. (B) Relative luciferase activity in HCT116 and LOVO cells co-transfected with miR-17-5p mimics together with reporter vectors carrying HSPB2 3'-UTR binding sites. (C) Relative luciferase activity in HCT116 and LOVO cells co-transfected with miR-17-5p inhibitor together with reporter vectors carrying HSPB2 3'-UTR binding sites. * $\mathrm{P}<0.05$; ** $\mathrm{P}<0.01$; *** $\mathrm{P}<0.001$. 
$\mathbf{a}$

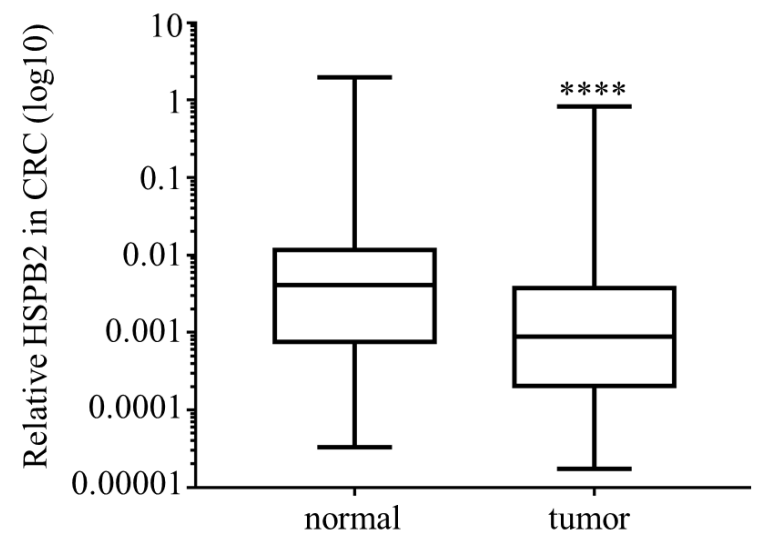

c

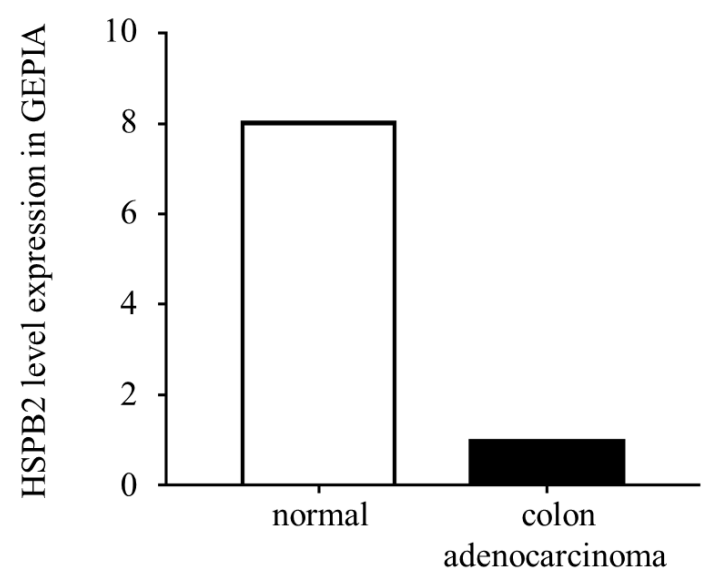

b

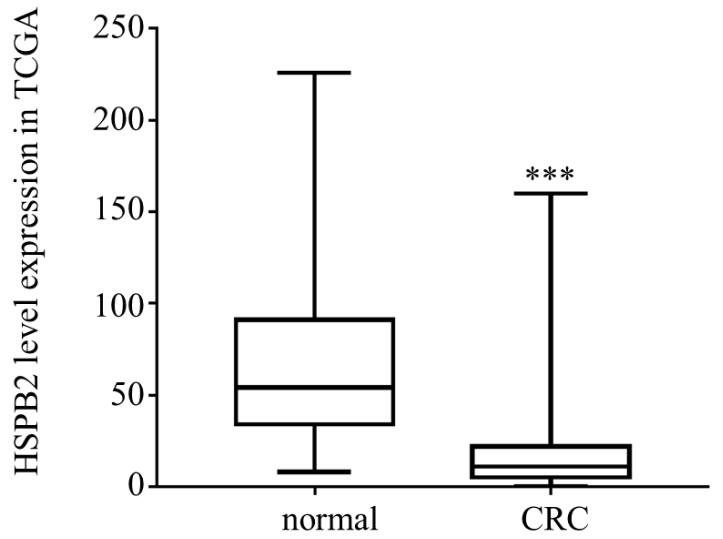

d

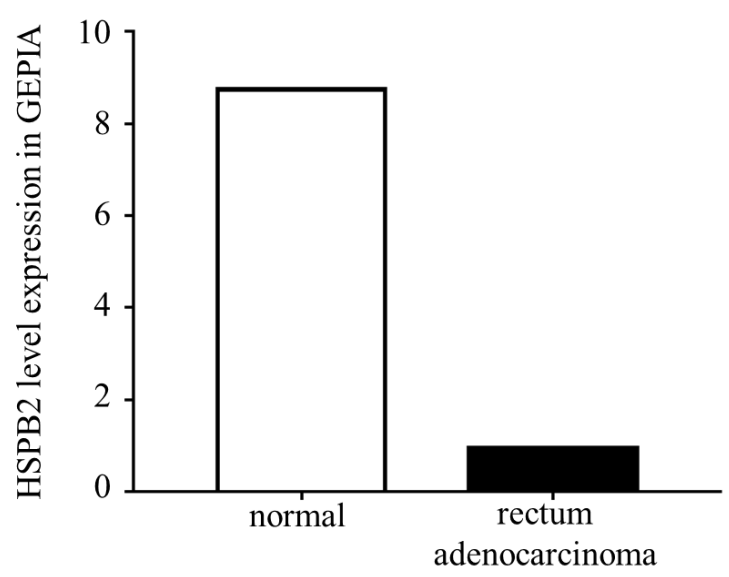

Figure 6

The expression of HSPB2 mRNA is down-regulated in CRC tissues. (A) The expression level of HSPB2 in 51 paired of CRC and adjacent normal tissues by qRT-PCR. (B) The HSPB2 expression profile of CRC tissues ( $n=647)$ and normal tissues $(n=51)$ in CRC dataset of TCGA. (C) The HSPB2 expression profile of colon adenocarcinoma tissues $(n=275)$ and normal tissues $(n=349)$ in COAD dataset of GEPIA. (D) The HSPB2 expression profile of rectum adenocarcinoma tissues $(n=92)$ and normal tissues $(n=318)$ in READ

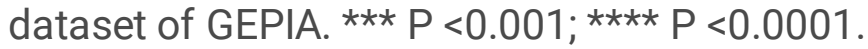


a

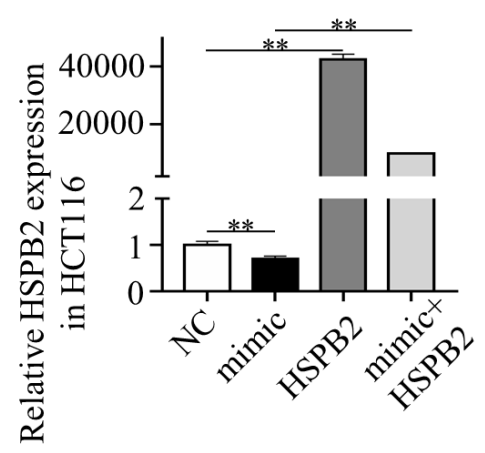

b
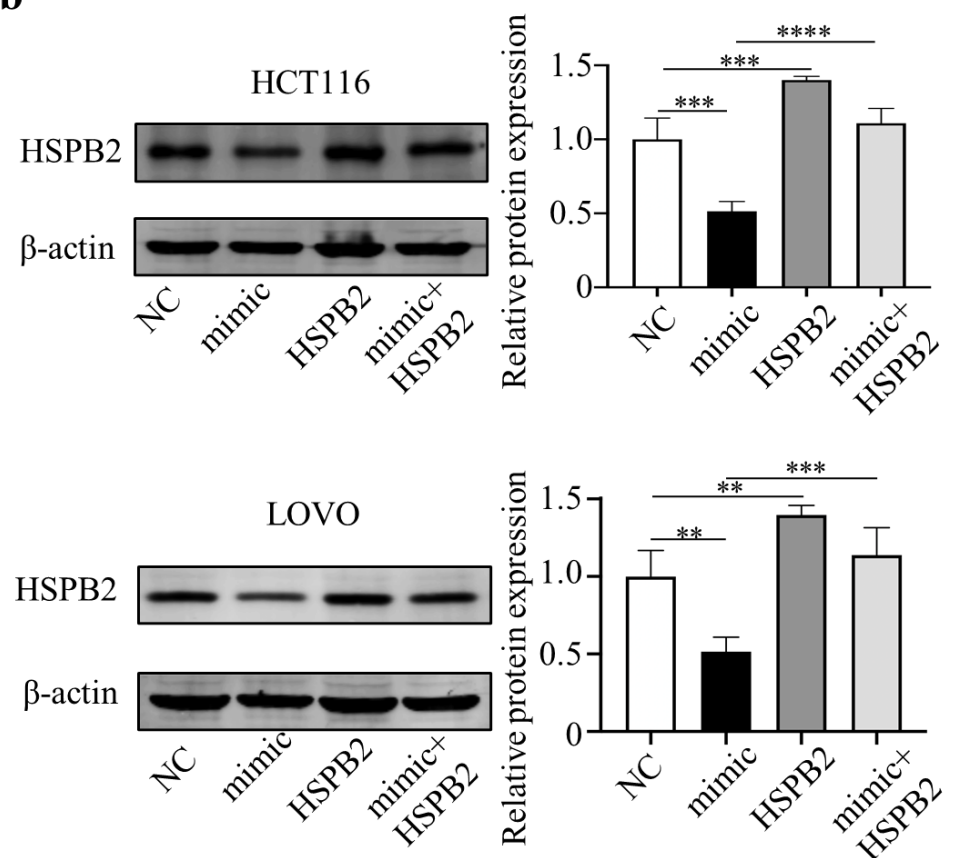
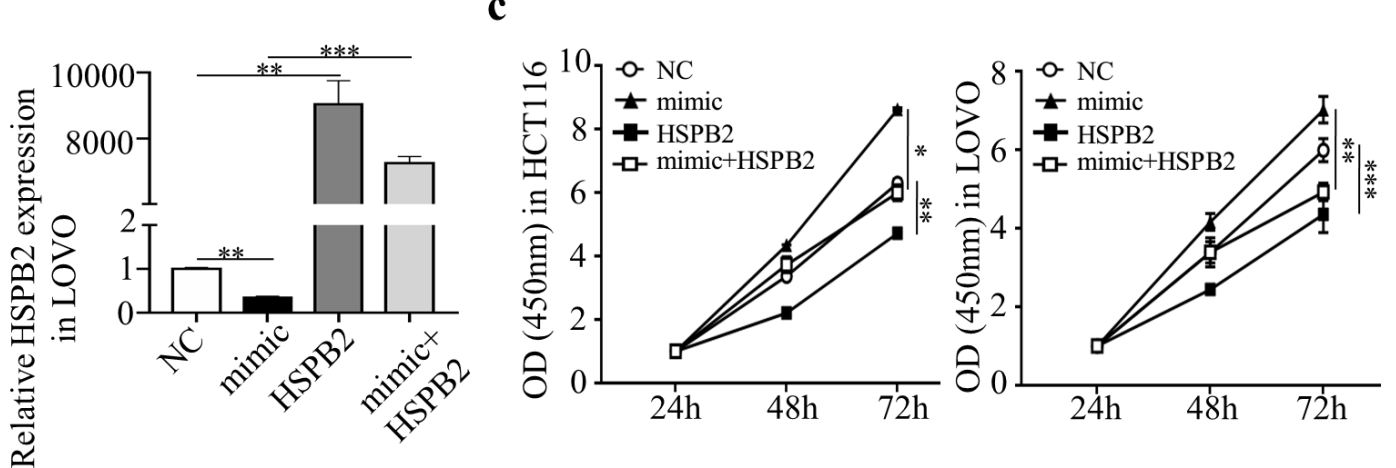

d
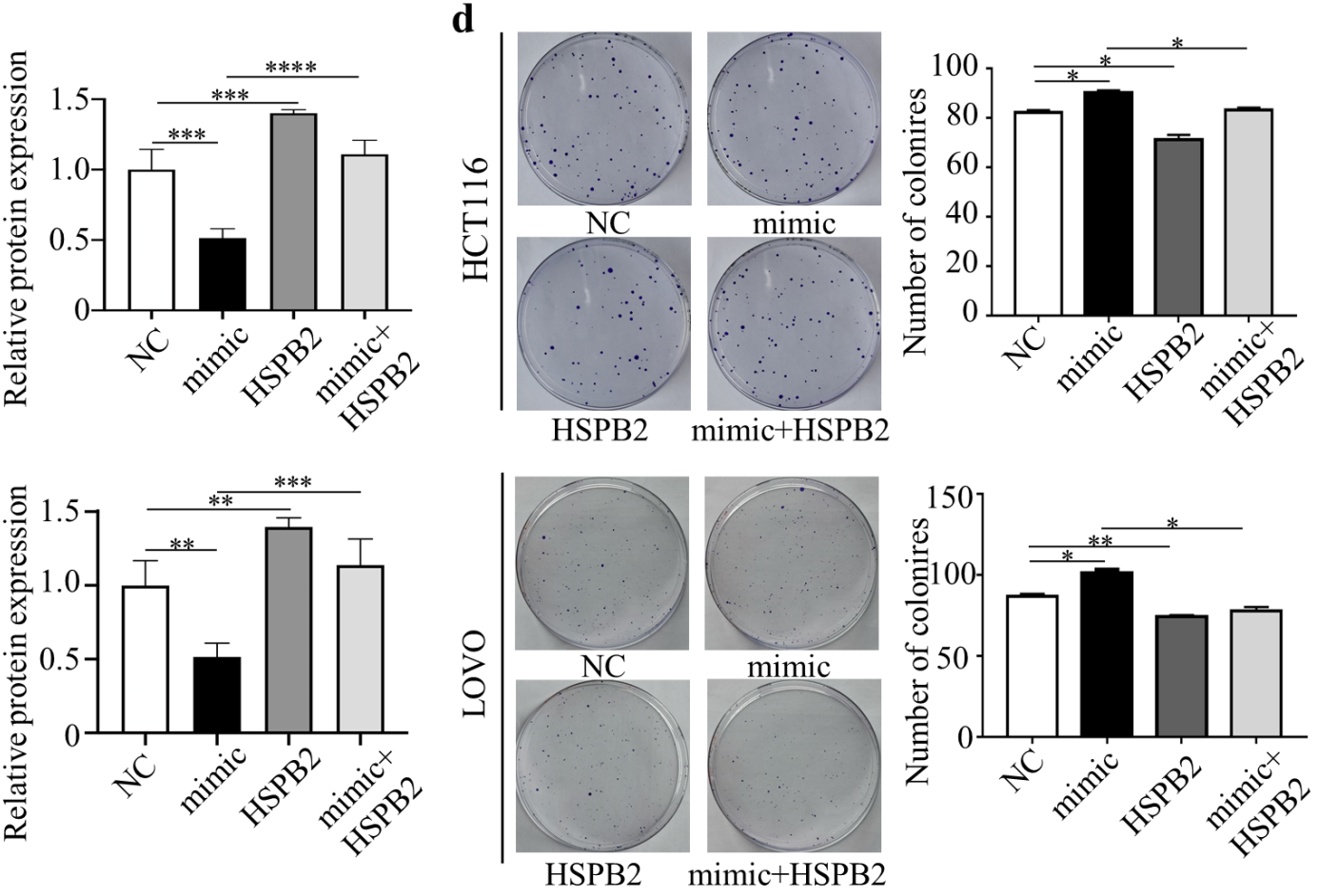

\section{Figure 7}

Reintroduction of HSPB2 could reverse the promotion of miR-17-5p in cell proliferation. (A, B) The overexpression of miR-17-5p and HSPB2 alone or simultaneously in HCT116 and LOVO cells confirmed by qRT-PCR and western blot analysis. (C, D) CCK8 analysis and colony formation assay were used to determine the effect of miR-17-5p and HSPB2 overexpression alone or simultaneously on cell

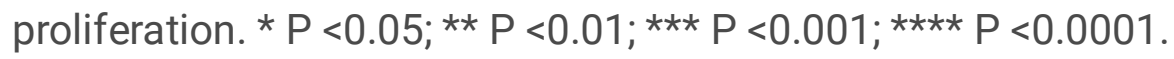



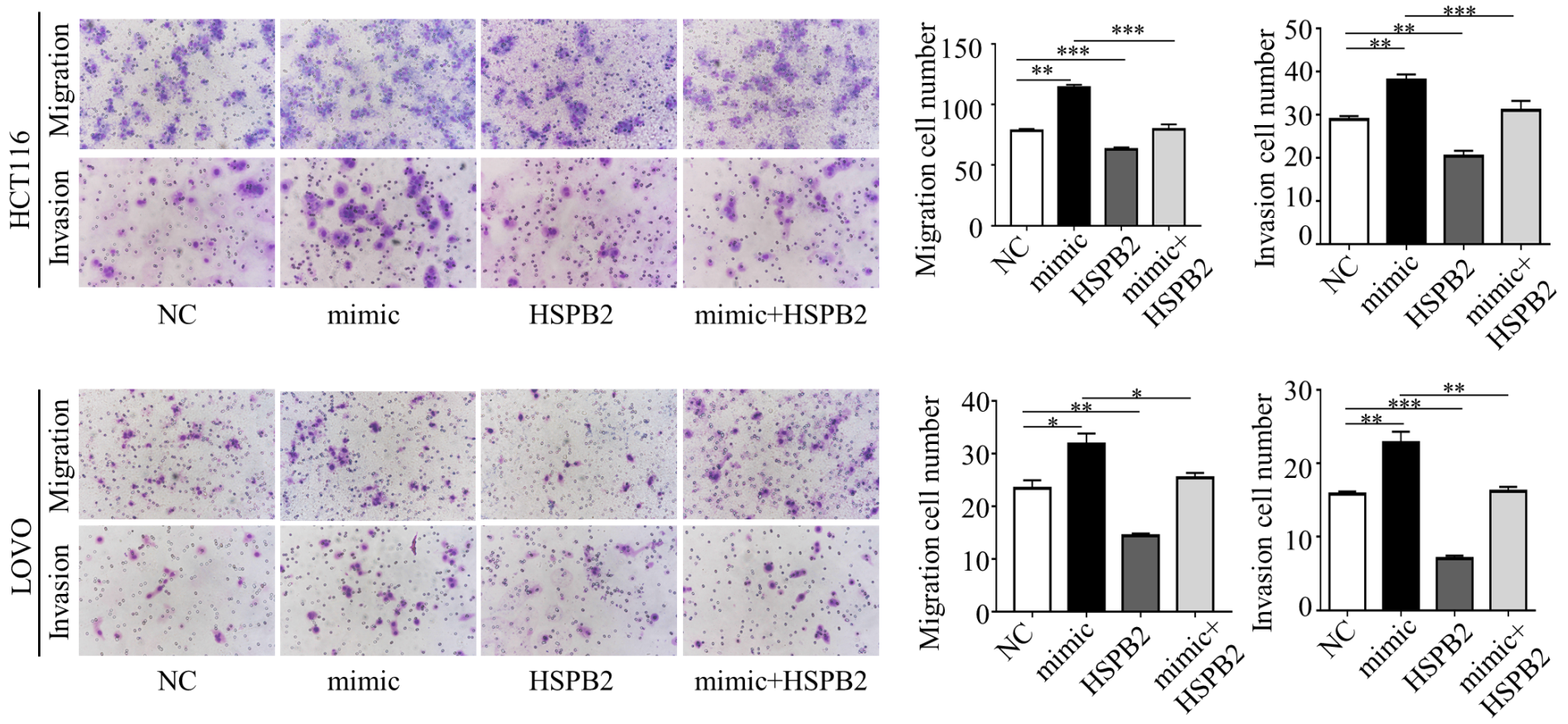

Figure 8

Transwell assay was performed to assess the migration and invasion potency after the expression of miR-17-5p and HSPB2 was up-regulated alone or simultaneously. ${ }^{*} P<0.05 ; * \star P<0.01 ; \star \star \star ~ P<0.001$. 
a

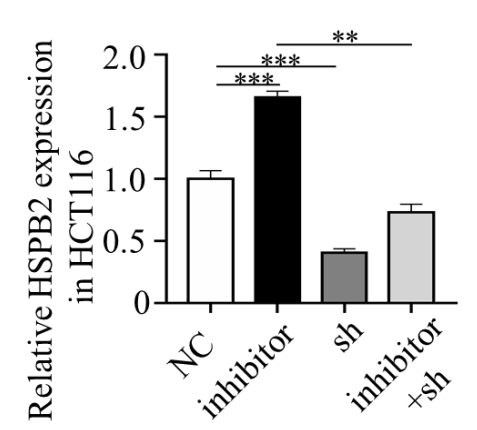

b
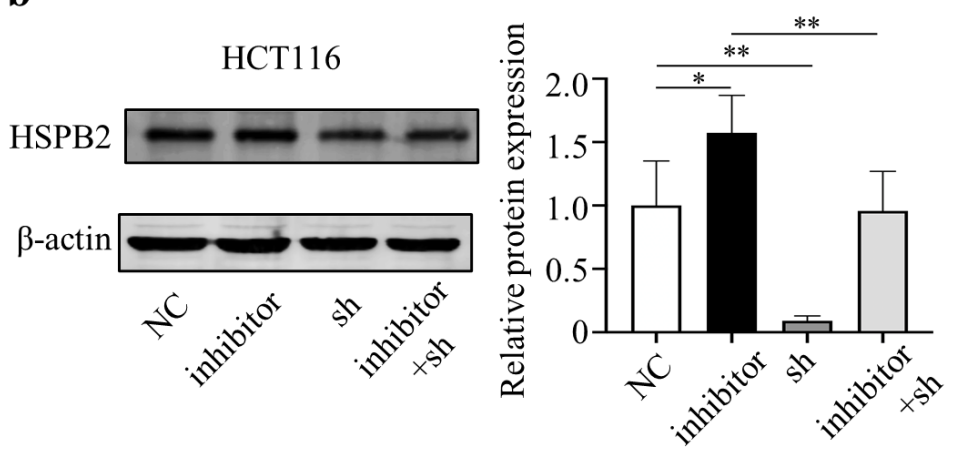

LOVO
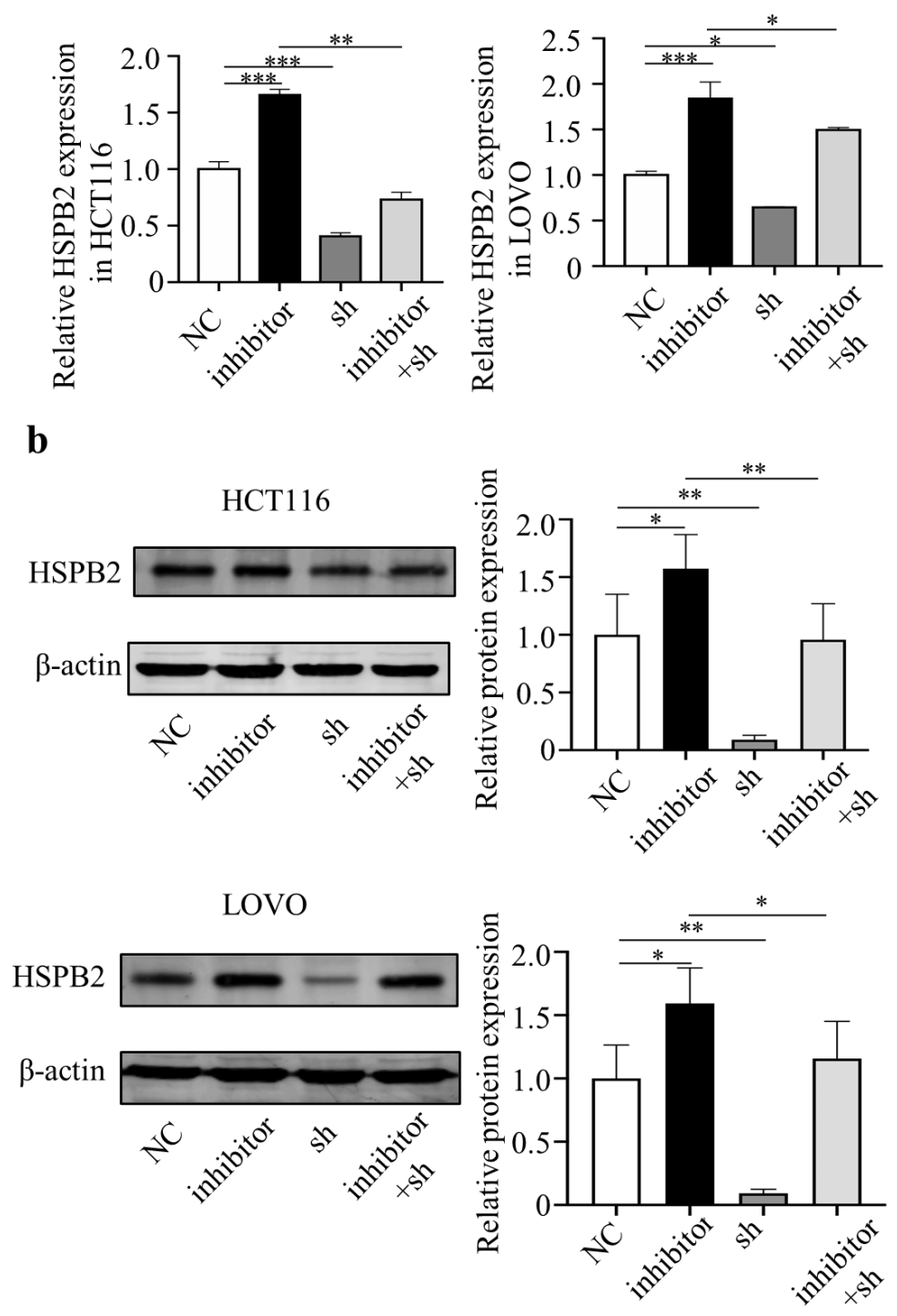

c
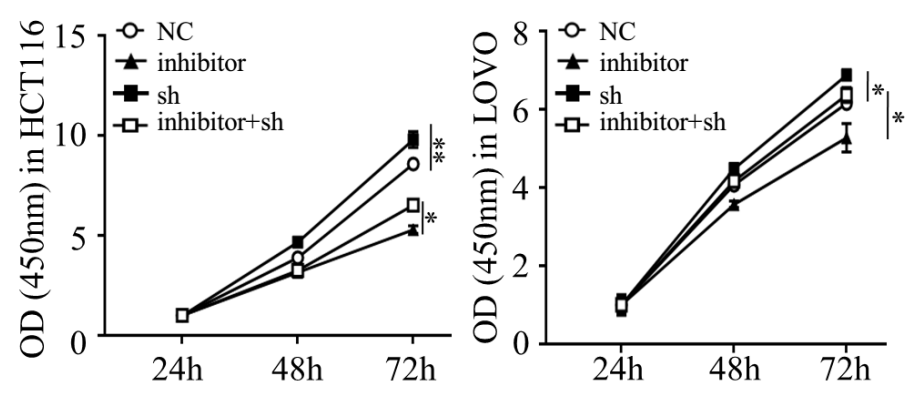

d
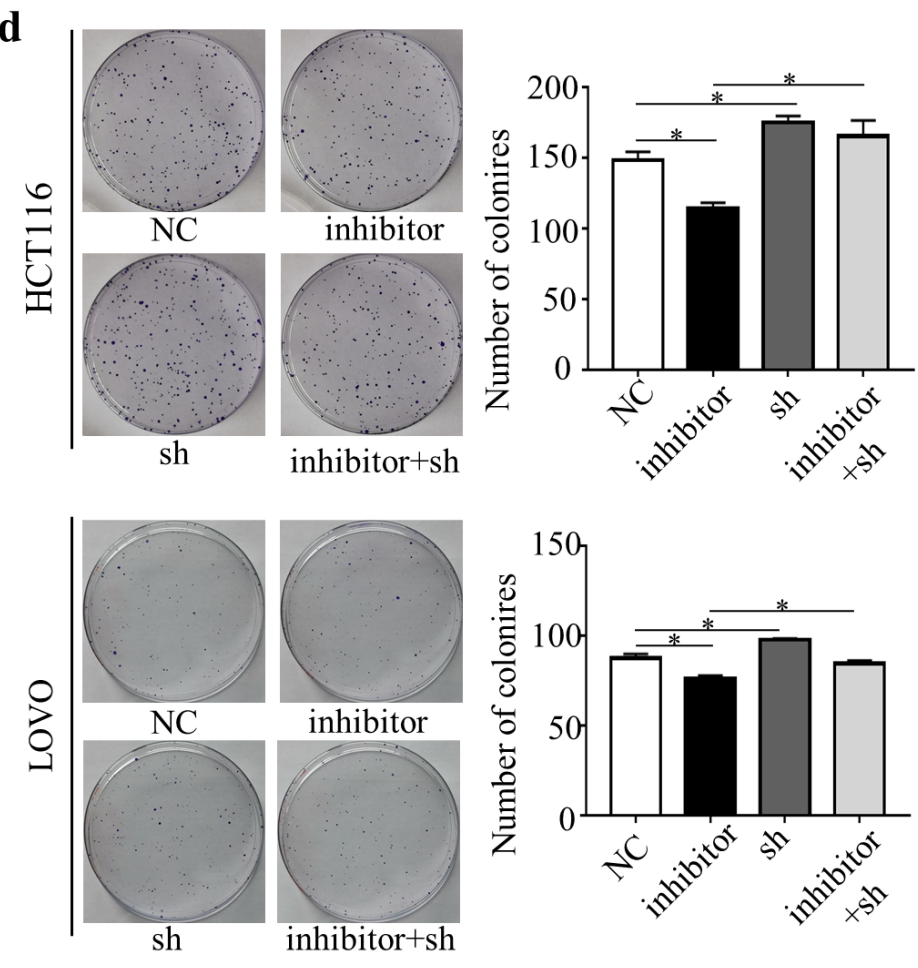

\section{Figure 9}

Reduced HSPB2 could rescue the suppression of miR-17-5p inhibitor in cell proliferation. (A, B) The decrease expression of miR-17-5p and HSPB2 alone or simultaneously in HCT116 and LOVO cells confirmed by qRT-PCR and western blot analysis. (C, D) CCK8 analysis and colony formation assay were used to determine the effect of knockdown miR-17-5p and HSPB2 alone or simultaneously on cell proliferation. * $\mathrm{P}<0.05 ; * \star \mathrm{P}<0.01 ; * \star * \mathrm{P}<0.001$. 

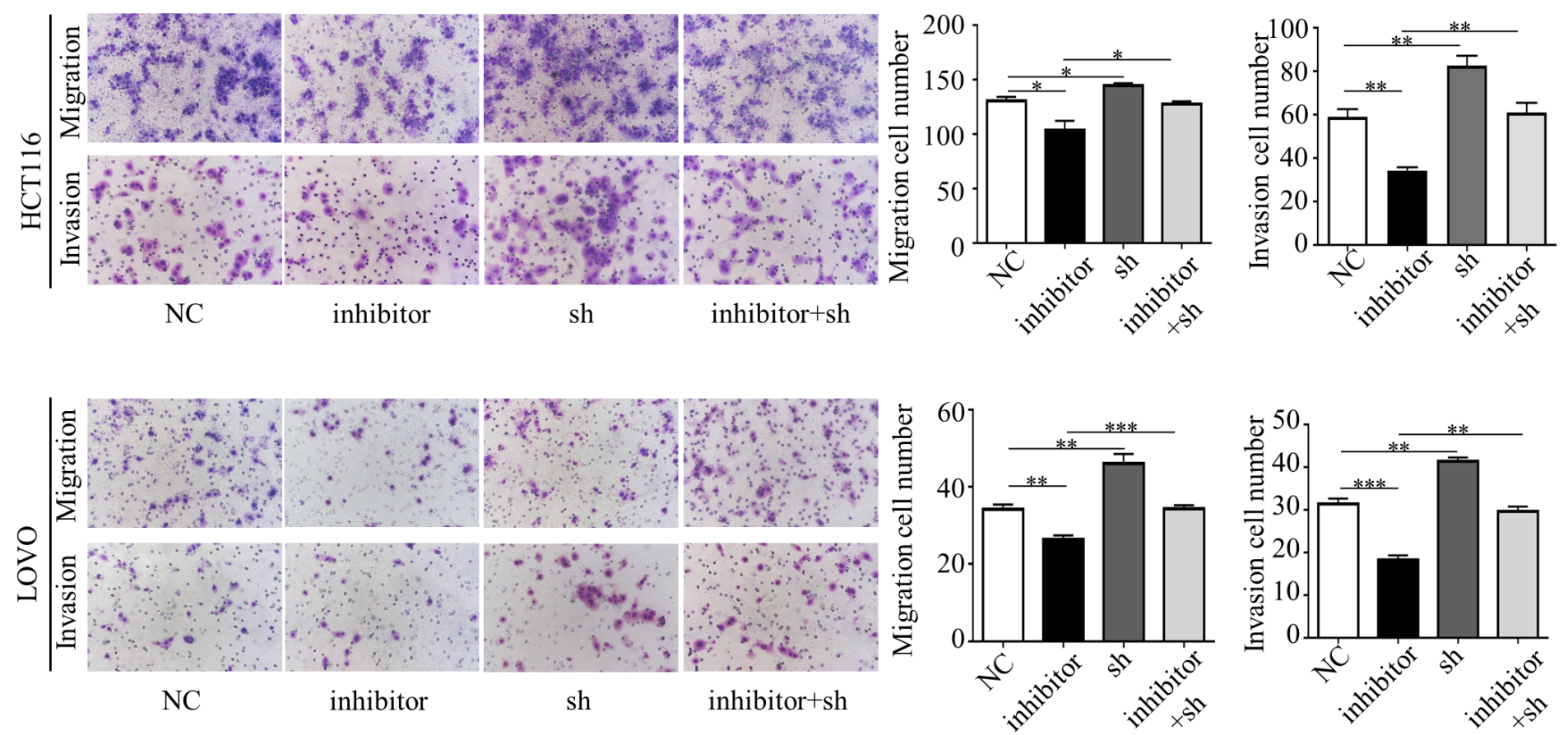

Figure 10

Transwell assay was performed to assess the migration and invasion potency after the expression of miR-17-5p and HSPB2 was down-regulated alone or simultaneously. ${ }^{*}<<0.05$; $* \star P<0.01$; $* \star * P<0.001$.

\section{Supplementary Files}

This is a list of supplementary files associated with this preprint. Click to download.

- SupplementaryMaterials.pdf 